Authors' accepted version for publication in Formal Aspects of Computing

The final publication is available at springerlink.com

\title{
The Behavioural Semantics of Event-B Refinement
}

\author{
Steve Schneider ${ }^{1}$, Helen Treharne ${ }^{1}$, Heike Wehrheim ${ }^{2}$ \\ 1 S.Schneider,H.Treharne\}@surrey.ac.uk, \\ Department of Computing, University of Surrey \\ 2 wehrheim@uni-paderborn.de \\ Department of Computer Science, University of Paderborn
}

\begin{abstract}
Event-B provides a flexible framework for stepwise system development via refinement. The framework supports steps for (a) refining events (one-by-one), (b) splitting events (one-by-many), and (c) introducing new events. In each of the steps events can be indicated as convergent (to be made internal) or anticipated (treatment deferred to a later refinement step). All such steps are accompanied with precise proof obligations. However, no behavioural semantics has been provided to validate the proof obligations, and no formal justification has previously been given for the application of these rules in a refinement chain. Behavioural semantics expresses a clear relationship between the first and last machines in a refinement chain. The framework we present provides a coherent justification for Abrial's approach to refinement in Event-B, and its generalisation to interface extension: adding events to the interface. In this paper, we give a behavioural semantics for Event-B refinement, with a treatment for the first time of splitting events and of anticipated events, adding to the well-understood treatment of convergent events. To this end, we define a CSP semantics for Event-B and show how the different forms of Event-B refinement can be captured as CSP refinement. It turns out that the appropriate CSP refinement relationship is influenced by the particular Event-B development strategy taken. We present two such strategies, one allowing, the other disallowing interface extensions.
\end{abstract}

Keywords: Event-B, CSP, refinement, traces, divergences, development strategy.

\section{Introduction}

Event-B [Abr10, MAV05] provides a framework for formal system development through stepwise refinement. Refinement allows to gradually build a complex system model by introducing more detail with every step. Individual refinement steps in Event-B are verified with respect to their proof obligations, and the transitivity of refinement ensures that the final system description is a refinement of the initial one. The refinement

Correspondence and offprint requests to: S. Schneider, H. Treharne, H. Wehrheim 
process allows new events to be introduced through the refinement process, in order to provide the more concrete implementation details necessary as refinement proceeds.

The framework allows for a great deal of flexibility to cover a broad range of system developments. The recent book [Abr10] comprising case studies from rather diverse areas shows that this goal is actually met. The basic modelling entity of Event-B are machines and their events. The flexibility of Event-B development a result of the different ways of dealing with events during refinement. At each step existing events of an Event-B machine need to be refined. This can be achieved by (a) simply keeping the event as is, (b) refining it into another event, possibly because of a change of the state variables, or (c) splitting it into several events ${ }^{1}$. Furthermore, every refinement step allows for the introduction of new events. To help reasoning about divergence, events are in addition classified as ordinary, anticipated or convergent. New events introduce new detail at the interface of a machine specification, and are introduced as anticipated or convergent. Convergent events are considered internal and therefore must not be executed forever. Their treatment within action systems refinement has been well established [But92]. For anticipated events the decision whether this is to be an internal or external event (i.e., part of the interface) is deferred to later refinement steps, but must be made by the end of the refinement chain. The use of anticipated events is a more recent development, introduced within the context of Event-B [Abr10, $\left.\mathrm{ABH}^{+} 10\right]$. Refinement steps come with precise proof obligations on all kinds of events; appropriate tool support helps in discharging these [BH07, ABHV08, $\left.\mathrm{ABH}^{+} 10\right]$. Event$\mathrm{B}$ is essentially a state-based specification technique like Z [WD96] or Alloy [Jac02], and proof obligations therefore reason about predicates on states.

However, no behavioural semantics has been provided to validate the proof obligations, and no formal justification has previously been given for the application of these rules in a refinement chain. In fact, it is hard to find one fixed formal semantics for Event-B at all. Instead, a recent article of Hallerstede [Hal11] advocates the absence of one fixed semantics as an advantage because it increases the flexibility of the specification formalism and allows to employ it in a variety of modelling domains - all with the same type of proof obligations. Nevertheless, even Hallerstede mentions that the design of Event-B has been inspired by action systems [BvW98], and Butler [But12] also implicitly uses an action system, i.e. weakest-precondition semantics for Event-B machines. Here we also follow this view on Event-B and treat events like guarded commands with a weakest-precondition semantics.

Our specific interest however is in refinement, and in particular in investigating the effect of the proof obligations on the various types of events with respect to the semantics. Our behavioural semantics expresses a clear relationship between the machines in a refinement chain, in particular the initial and the final one. The framework we present provides a coherent justification for Abrial's approach to refinement in Event-B, and its generalisation to interface extension: adding events to the interface. Our framework is based on the process algebra CSP which provides us - besides a sound and large body of underlying theory — with a clear notion of refinement and a number of operators for dealing with specific sets of events in refinement (e.g. hiding).

In order to understand the relationship between Event-B and CSP refinement, these two concepts need to be set in a single framework. Both formalisms support a variety of different forms of refinement: Event-B by means of several proof obligations related to refinement, out of which the system designer chooses an appropriate set; CSP by means of its different semantic domains of traces, failures and divergences. The aim of this paper is to give a behavioural semantics for Event-B refinement in terms of CSP's behaviouroriented process refinement[Hoa85]. This will also provide the underlying results that support refinement in the combined formalism Event-B\|CSP [STW10]. It turns out that CSP supports an approach to refinement consistent with that of Event-B. It faithfully reflects all of Event-B's possibilities for refinement, including splitting events and new events. It also deals with the Event-B approach of anticipated events as a means to defer consideration of divergence-freedom. Our results involve support for individual refinement steps as well as additional results for the resulting refinement chain. Our work is thus in line with previous studies relating state-based with behaviour-oriented refinement (see e.g. [BD02, DB03, BD09]).

It turns out that our results closely depend on the development strategy chosen for the refinements. Development strategies impose rules on the use of different types of events and their refinements. To the best of our knowledge development strategies for Event-B have not been discussed so far, let alone formalised. Tools supporting development and proof of Event-B designs like Rodin $\left[\mathrm{ABH}^{+} 10\right]$ only give very weak restrictions on the use of event types. Thus another contribution of this paper is the formal definition of

$\overline{1}$ A fourth option is merging of events which we do not consider here. 
two different development strategies for Event-B, depending on whether or not to enable interface extension: where the machine interface (akin to an API) is extended to allow additional events. Basically, strategy I disallows interface extensions whereas strategy II allows them. This means that with strategy I the interface of a system is given by the initial machine specification, and all events introduced during refinement steps are eventually hidden. This is the approach taken in [Abr10]. Strategy II is the more general one, allowing for a greater flexibility in development, however, at the price of achieving a weaker relationship between the machines in a refinement. Strategy II is the approach supported by Rodin [EB11]. For both strategies we have identified results about the corresponding CSP refinement. Refinement in Event-B does not require liveness properties to be preserved. We therefore do not consider them in this paper.

The paper is structured as follows. The next section introduces the necessary background on Event-B and CSP, and also introduces the first part of our running example. Section 3 gives the CSP semantics for Event$\mathrm{B}$ based on weakest preconditions. In Section 4 we precisely fix the notion of refinement used in this paper, both for CSP and for Event-B, and further develop our example. Section 5 then sets the two refinement definitions in relation, first of all just for one step in a refinement and independent of the strategy used for chains of refinement. It turns out that the appropriate refinement concept of CSP that underpins Event-B is infinite-traces-divergences refinement. Sections 6 and 7 then present a formal definition of the two strategies I and II and set these in relation with a CSP refinement concept for chains of refinements. The last section concludes.

This paper is an extension of [STW11b] introducing a new refinement strategy, with its associated definitions and new results, and including a larger example used throughout the whole paper for both strategies.

\section{Background}

We start with a short introduction to Event-B and CSP. For more detailed information see [Abr10] and [Sch99] respectively.

\subsection{Event-B}

Event-B [Abr10, MAV05] is a state-based specification formalism based on set theory. The basic modelling entity in Event-B are machines with their events. Here we describe the basic elements of an Event-B machine required for this paper; a full description of the formalism can be found in [Abr10].

The main modelling construct in Event-B is a machine, which encapsulates state and events which can query or update the state. A machine specification describes state information in terms of state variables and invariants on them. The machine describes how the state is initialised, and also describes guarded events, which describe how and when the state may be updated. These are the core elements of Event-B that we are concerned with in this paper.

Event-B machines are structured into a number of clauses which are concerned with different aspects of the specification. Here we concentrate on those clauses concerned with state and events. We will therefore describe a machine $M$ with a list of state variables $v$, a state invariant $I(v)$, and a set of events $e v, \ldots$ to update the state (see left of Figure1). Initialisation is a special event init which sets the initial state of the machine, and its guard is true.

Event-B also in general allows sets which introduce new types, and constants. However, for our purposes the treatment of elements such as sets and constants are independent of the results of this paper, and so we will not include them here. However, they can be directly incorporated without affecting our results.

A machine $M$ will have various proof obligations on it. These include consistency obligations: that events preserve the invariant. They can also include (optional) deadlock-freeness obligations: that at least one event guard is always true.

Central to an Event-B description is the definition of the events, each consisting of a guard $G(v)$ over the variables, and a body, usually written as an assignment $S$ on the variables. The body defines a before-after predicate $B A\left(v, v^{\prime}\right)$ describing changes of variables upon event execution, in terms of the relationship between the variable values before $(v)$ and after $\left(v^{\prime}\right)$. The body can also be written as $v: \mid B A\left(v, v^{\prime}\right)$, whose execution assigns to $v$ any value $v^{\prime}$ which makes the predicate $B A\left(v, v^{\prime}\right)$ true (see right of Fig. 1 , where $B A$ is the predicate in event $e v t$ ). In Event-B an event may also introduce local variables, which can be included in 


\begin{tabular}{|lc|}
\hline machine $M$ & $e v \widehat{=}$ \\
variables $v$ & when \\
invariant $I(v)$ & $G(v)$ \\
events init, ev, .. & then \\
end & end $: \mid B A\left(v, v^{\prime}\right)$ \\
\hline
\end{tabular}

Fig. 1. Template of an Event-B machine and an event.

the guard (which constrains what values they can take), and in the body where they can have an effect on the change of state. Such events are constructed as:

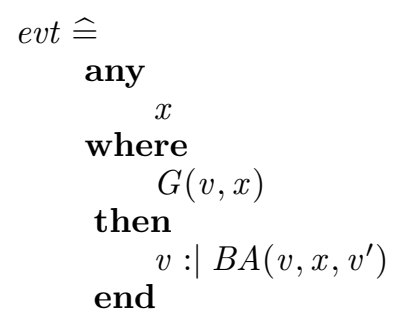

For our purposes, the treatment of local variables is orthogonal to our results, and so we do not consider them here. They can be directly incorporated without affecting our results. We will thus use the form of events presented in Fig. 1.

The Event-B approach to semantics, provided in [Abr10, MAV05], is to associate proof obligations with machines. The key proof obligation on events is that they preserve the invariant: when an event is called within its guard, then the state resulting from executing the body should meet the invariant. For example, in the case of the machine in Fig. 1 (which does not include sets or constants) we obtain the following proof obligation INV on events which have the form of evt:

$$
\begin{array}{|l|l|}
\hline \begin{array}{l|}
I(v) \wedge G(v) \wedge B A\left(v, v^{\prime}\right) \\
I\left(v^{\prime}\right)
\end{array} & \text { INV } \\
\hline
\end{array}
$$

This is a particular case of the INV rule given in [Abr10, page 189]. Discharging this proof obligation establishes that the event preserves the invariant, and so is well-defined.

There is no definitive presentation of the core proof obligations that any machine must satisfy, but the proof obligations presented in [Abr10, MAV05] provide the key rules. In practice, the proof obligations generated by the Rodin platform $\left[\mathrm{ABH}^{+} 10\right]$ give the de facto rules for a machine.

In this paper we will make use of weakest precondition semantics for guarded commands, applying them to B events. We introduce this semantics in Section 3. This semantics gives a similar treatment of events as the proof obligations approach of Event-B, but from the point of view of considering an event as defining a relation between before- and after-states. The proof obligations approach is geared towards verification and is directly suited to the tool support.

As an example of an Event-B machine, consider the machine given in Fig. 2 abstractly specifying the (protocol of use of a) basket in a store. The machine represents the initial step of a development. In later sections we will gradually refine this machine, and by this introduce the concept of refinement in Event-B; in particular the different kinds of events occurring during refinement.

The machine defines a basket to be in one of two possible states: either empty or complete. Complete corresponds to the case when the customer has decided to have completed his shopping. The notation for the events is a short version of the above given as the guard for the events is true and the before-after-predicate allows for a single after value only, thus the event is simply written as an assignment. The two events (which are both unguarded and thus can always be executed) do the checkout and empty the basket, respectively. In addition, the machine has an event init which is however omitted since it is empty. 


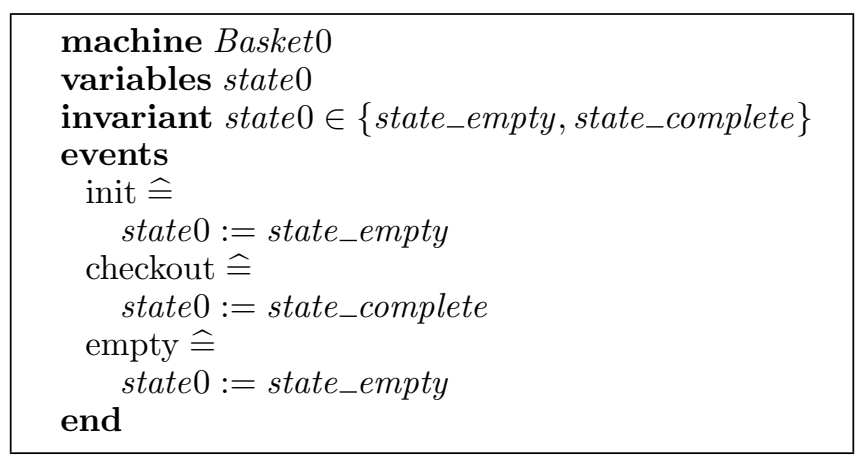

Fig. 2. Event-B machine Basket0

\subsection{CSP}

CSP, Communicating Sequential Processes, introduced by Hoare [Hoa85] is a formal specification language aiming at the description of communicating processes. A process is characterised by the events it can engage in and their ordering. Events will in the following be denoted by $a_{1}, a_{2}, \ldots$. The set of all possible events is denoted $\Sigma$. Process expressions are built out of events using a number of composition operators. In this paper, we will make use of just three of them: interleaving $\left(P_{1} \| \mid P_{2}\right)$, executing two processes in parallel without any synchronisation; hiding $(P \backslash N)$, making a set $N$ of events internal; and renaming $(f(P)$ and $f^{-1}(P)$ ), changing the names of events according to a renaming function $f$. If $f$ is a non-injective function, $f^{-1}(P)$ will offer a choice of events $b$ such that $f(b)=a$ whenever $P$ offers event $a$.

Every CSP process $P$ has an alphabet $\alpha P$. Its semantics is given using the Failures/Divergences/Infinite Traces semantic model for CSP. This is presented as $\mathcal{U}$ in [Ros98] or FDI in [Sch99]. The semantics of a process can be understood in terms of four sets, $T, F, D, I$, which are respectively the (finite) traces, failures, divergences, and infinite traces of $P$. These are understood as observations of possible executions of the process $P$, in terms of the events from $\alpha P$ that it can engage in.

Traces are finite sequences of events from $P$ 's alphabet: $\operatorname{tr} \in \alpha P^{*}$. The set traces $(P)$ represents the possible finite sequences of events that $P$ can perform. Since this paper is not addressing liveness, failures will not need to be considered in this paper and are therefore not explained here.

Divergences are finite sequences of events on which the process might diverge: perform an infinite sequence of internal events (such as an infinite loop) at some point during or at the end of the sequence. After a process has diverged, the semantics allows any events from $\Sigma$ to be possible. The set divergences $(P)$ is the set of all possible divergences for $P$. Infinite traces $u \in \alpha P^{\omega}$ are infinite sequences of events. The set infinites $(P)$ is the set of infinite traces that $P$ can exhibit. For technical reasons it also contains those infinite sequences that have some prefix which is a divergence.

Definition 2.1. A process $P$ is divergence-free if divergences $(P)=\{\}$.

We use $t r$ to refer to finite traces. These can also be written explicitly as $\left\langle a_{1}, a_{2}, \ldots, a_{n}\right\rangle$. The empty trace is \langle\rangle , concatenation of traces is written as $t r_{1} \frown t r_{2}$. We use $u$ to refer to infinite traces. Given a set of events $A$, the projections $\operatorname{tr}\lceil A$ and $u\lceil A$ are the traces restricted to only those events in $A$. Note that $u \uparrow A$ might be finite, if $A$ events appear only finitely many times in $u$. Conversely, $\operatorname{tr} \backslash A$ and $u \backslash A$ are those traces with the events in $A$ removed. The length operator \#tr and \# $u$ gives the length of the trace it is applied to which can be an integer or infinity. The alphabet operator $\alpha t r$ and $\alpha u$ gives us the alphabet of a trace, i.e., the set of events occuring in it. Given two traces $t r_{1}$ and $t r_{2}, t r_{1}|| t r_{2}$ defines the set of all traces presenting interleavings of the first and the second trace, i.e., combinations which keeps the ordering of the original traces but arbitrarly mix elements from the first with the second trace. Finally, given a renaming function $f$ on the set of events, $f(t r)$ is the trace in which all elements are renamed according to $f$. Interleaving and renaming are also defined in a similar way on infinite traces. As an example for all these definitions consider the following: 


$$
\begin{aligned}
& \langle a, b\rangle \frown\langle c, b, c\rangle=\langle a, b, c, b, c\rangle \\
& \langle a, b, a, c, c, c, b\rangle \uparrow\{a, b\}=\langle a, b, a, b\rangle \\
& \langle a, b, a, c, c, c, b\rangle \backslash\{a, b\}=\langle c, c, c\rangle \\
& \#\langle a, b, a, c, c, c, b\rangle=7 \\
& \langle a, c, d, b\rangle \in(\langle a, b\rangle \|\langle c, d\rangle) \\
& \langle a, d, c, b\rangle \notin(\langle a, b\rangle \|\langle c, d\rangle) \\
& f(\langle a, b, b\rangle)=\langle c, d, d\rangle \text { for } f:\{a \mapsto c, b \mapsto d\}
\end{aligned}
$$

Such operations on traces are, for instance, used to define the semantics of the process composition operators in CSP, like hiding, interleaving and renaming explained above. Since we make use of them later, we will give the formal definitions for the infinite traces and divergences semantics here.

Definition 2.2. Let $P$ be a process, $A$ a set of events, $f$ a renaming function.

$$
\begin{aligned}
& \operatorname{divergences}(P \backslash A)=\left\{(\operatorname{tr} \backslash A) \frown t r^{\prime} \mid \operatorname{tr} \in \operatorname{divergences}(P)\right\} \\
& \cup\left\{(u \backslash A) \frown \operatorname{tr}^{\prime} \mid u \in \text { infinites }(P) \wedge \#(u \backslash A)<\infty\right\} \\
& \text { infinites }(P \backslash A)=\{u \backslash A \mid u \in \text { infinites }(P) \wedge \#(u \backslash A)=\infty\} \\
& \cup\left\{\operatorname{tr} \frown u^{\prime} \mid t r \in \operatorname{divergences}(P \backslash A)\right\} \\
& \operatorname{divergences}(P \| Q)=\left\{t r \frown t r^{\prime} \mid t r \in\left(\operatorname{tr}_{P} \mid \| \operatorname{tr}_{Q}\right), \operatorname{tr}_{P} \in \operatorname{divergences}(P) \wedge t r_{Q} \in \operatorname{traces}(Q)\right\} \\
& \cup\left\{t r \frown \operatorname{tr}^{\prime} \mid \operatorname{tr} \in\left(\operatorname{tr}_{P} \| \operatorname{tr}_{Q}\right), \operatorname{tr}_{P} \in \operatorname{traces}(P) \wedge \operatorname{tr}_{Q} \in \operatorname{divergences}(Q)\right\} \\
& \text { infinites }(P \| Q)=\left\{u \mid u \in\left(u_{P} \| u_{Q}\right), u_{P} \in \operatorname{infinites}(P) \wedge u_{Q} \in \operatorname{infinites}(Q)\right\} \\
& \cup\left\{u \mid u \in\left(u_{P} \mid \| \operatorname{tr}_{Q}\right), u_{P} \in \operatorname{infinites}(P) \wedge \operatorname{tr}_{Q} \in \operatorname{traces}(Q)\right\} \\
& \cup\left\{u \mid u \in\left(\operatorname{tr}_{P}|| u_{Q}\right), \operatorname{tr}_{P} \in \operatorname{traces}(P) \wedge u_{Q} \in \operatorname{infinites}(Q)\right\} \\
& \cup\left\{\operatorname{tr} \frown u^{\prime} \mid \operatorname{tr} \in \operatorname{divergences}(P \| Q)\right\} \\
& \text { divergences }(f(P))=\left\{f(t r) \frown t r^{\prime} \mid t r \in \text { divergences }(P)\right\} \\
& \text { infinites }(f(P))=\{f(u) \mid u \in \text { infinites }(P)\} \\
& \cup\left\{\operatorname{tr} \frown u^{\prime} \mid \operatorname{tr} \in \text { divergences }(f(P))\right\} \\
& \text { divergences }\left(f^{-1}(P)\right)=\left\{t r \frown t r^{\prime} \mid f(t r) \in \operatorname{divergences}(P)\right\} \\
& \text { infinites }\left(f^{-1}(P)\right)=\{u \mid f(u) \in \text { infinites }(P)\} \\
& \cup\left\{\operatorname{tr}^{\frown} u^{\prime} \mid \operatorname{tr} \in \text { divergences }(f(P))\right\}
\end{aligned}
$$

In this definition, $t r^{\prime}$ ranges over finite sequences of events from $\Sigma$, and $u^{\prime}$ ranges over infinite sequences of events from $\Sigma$.

As a first observation on infinite traces, hiding and divergences, we get the following:

Lemma 2.3. If $P$ is divergence-free, and for every infinite trace $u$ of $P$ we have $\#(u \backslash A)=\infty$, then $P \backslash A$ is divergence-free.

Proof: Follows immediately from the semantics of the hiding operator.

Later, we furthermore use specifications on traces or, more generally, on CSP processes. Specifications are given in terms of predicates. If $S P E C$ is a predicate on a particular semantic element, then we write $P$ sat $S P E C$ to denote that all relevant elements in the semantics of $P$ meet the predicate $S P E C$. For example, if $S P E C(u)$ is a predicate on infinite traces, then $P$ sat $S P E C(u)$ is equivalent to $\forall u \in$ infinites $(P)$. SPEC $(u)$.

\section{CSP semantics for Event-B machines}

The development of Event-B, in particular of its notion of refinement, has been largely inspired by action systems [Hal11]. Thus Morgan's CSP semantics for action systems [Mor90] allows traces, failures, and di- 
vergences to be defined for Event-B machines, in terms of the sequences of events they can and cannot engage in. Butler's extension to handle unbounded nondeterminism [But92] defines the infinite traces for action systems. These together give a way of considering Event-B machines as CSP processes, and treating them within the CSP semantic framework. Note that the notion of traces for machines is different to that presented in [Abr10], where traces are considered as sequences of states rather than our treatment of traces as sequences of events. In this paper we use the infinite traces model in order to give a proper treatment of divergence under hiding. This is required to establish our main result concerning divergence-freedom under hiding of new events. Consideration of finite traces alone is not sufficient for this result.

More specifically, infinite traces need to be explicitly recorded since in the presence of unbounded nondeterminism they cannot be derived from the finite traces alone. Event-B easily allows for specifying unbounded nondeterminism. For this, consider for instance a machine initially choosing a value of some variable $n$ from $N A T$ and then executing an event $e v n$ times. This machine has no infinite trace of $e v$ events, but finite traces of every length $n$. We need to distinguish this machine from a machine which simply executes $e v$ forever since when hiding this event one machine will have divergent behaviour while the other does not.

The CSP semantics is based on the weakest-precondition semantics of events. Let $S$ be a statement (of an event). Then, following the notation of [Abr05] we will use $[S] R$ to denote the weakest precondition for statement $S$ to establish postcondition $R$. Weakest preconditions for events of the form when $G(v)$ then $S(v)$ end are given using Morgan's semantics for guarded commands [Mor88]:

[ when $G(v)$ then $S(v)$ end $] P=G(v) \Rightarrow[S(v)] P$

An event when $G(v)$ then $S(v)$ end executes $S(v)$ when the guard $G(v)$ is true, but it is blocked and cannot execute when the guard is false. Intuitively, [ when $G(v)$ then $S(v)$ end $] P$ is true in a state when every execution of the event from that state is guaranteed to terminate and reach a state in which $P$ holds. Hence when $G(v)$ is true we require that $[S(v)] P$ holds, that every execution of $S(v)$ is guaranteed to terminate and reach a state in which $P$ holds. Conversely, when $G(v)$ is false then it is vacuously true that every execution of when $G(v)$ then $S(v)$ end terminates and reaches a state where $P$ holds, since there are no such executions.

Similarly, events in the general form when $G(v)$ then $v: \mid B A\left(v, v^{\prime}\right)$ end have a weakest-precondition semantics as follows:

[ when $G(v)$ then $v: \mid B A\left(v, v^{\prime}\right)$ end $] P=G(v) \Rightarrow \forall v^{\prime} .\left(B A\left(v, v^{\prime}\right) \Rightarrow P\left[v^{\prime} / v\right]\right)$

Observe that for the case $P=$ true we have

[ when $G(v)$ then $v: \mid B A\left(v, v^{\prime}\right)$ end ] true $=$ true

Weakest preconditions and proof obligations are closely connected, and proof obligations associated with machines can also be expressed and understood in weakest-precondition terms. For example the requirement that an event $e v$ should preserve the invariant $I$ is naturally expressed as

$$
I \Rightarrow[e v] I
$$

This states that if the invariant $I$ holds for an initial state then performing $e v$ from that state should reach a final state where $I$ again holds. Applying the weakest precondition semantics for an event of the form when $G(v)$ then $v: \mid B A\left(v, v^{\prime}\right)$ end we obtain the requirement

$$
I(v) \Rightarrow\left[\text { when } G(v) \text { then } v: \mid B A\left(v, v^{\prime}\right) \text { end }\right] I(v)
$$

This reduces to the equivalent requirement (where $v$ and $v^{\prime}$ are both under an implicit universal quantification):

$$
\left(I(v) \wedge G(v) \wedge B A\left(v, v^{\prime}\right) \Rightarrow I\left(v^{\prime}\right)\right)
$$

Based on the weakest precondition semantics of [Mor90, But92] for action systems, we can define the traces, divergences and infinite traces of an Event-B machine ${ }^{2}$.

Traces The traces of a machine $M$ are those sequences of events $t r=\left\langle a_{1}, \ldots, a_{n}\right\rangle$ which are possible for $M$ (after initialisation init): those that do not establish false:

$$
\operatorname{traces}(M)=\{\operatorname{tr} \mid \neg[\text { init; } \operatorname{tr}] \text { false }\}
$$

$\overline{2}$ Failures can be defined as well but are omitted since they are not needed for our approach. 
Here, the weakest precondition on a sequence of events is the weakest precondition of the sequential composition of those events: $\left[\left\langle a_{1}, \ldots, a_{n}\right\rangle\right] P$ is given as $\left[a_{1} ; \ldots ; a_{n}\right] P=\left[a_{1}\right]\left(\ldots\left(\left[a_{n}\right] P\right) \ldots\right)$.

Divergences A sequence of events $t r$ is a divergence if the sequence of events is not guaranteed to terminate, i.e., $\neg[$ init; tr $]$ true. Thus

$$
\text { divergences }(M)=\{\operatorname{tr} \mid \neg[\text { init; } \operatorname{tr}] \text { true }\}
$$

Note that any Event-B machine $M$ with events of the form $e v$ given in Fig. 1 is divergence-free. This is because $[e v]$ true $=$ true for such events (and for init), and so $[$ init; tr $]$ true $=$ true. Thus no potential divergence $t r$ meets the condition $\neg[$ init; tr $]$ true.

Infinite Traces The technical definition of infinite traces is given in [But92], in terms of least fixed points of predicate transformers on infinite vectors of predicates. Informally, an infinite sequence of events $u=\left\langle u_{0}, u_{1}, \ldots\right\rangle$ is an infinite trace of $M$ if there is an infinite sequence of predicates $P_{i}$ such that $\neg[$ init $]\left(\neg P_{0}\right)$ (i.e., some execution of $i n i t$ reaches a state where $P_{0}$ holds), and $P_{i} \Rightarrow \neg\left[u_{i}\right]\left(\neg P_{i+1}\right)$ for each $i$ (i.e., if $P_{i}$ holds then some execution of $u_{i}$ can reach a state where $P_{i+1}$ holds).

$$
\begin{array}{ll}
\text { infinites }(M)=\left\{u \mid \text { there is a sequence }\left\langle P_{i}\right\rangle_{i \in \mathbb{N}},\right. & \neg[\text { init }]\left(\neg P_{0}\right) \wedge \\
& \text { for all } \left.i . P_{i} \Rightarrow \neg\left[u_{i}\right]\left(\neg P_{i+1}\right)\right\}
\end{array}
$$

These definitions give the CSP Traces/Divergences/Infinite Traces semantic model of Event-B machines in terms of the weakest-precondition semantics of events. For the above given Event-B machine Basket 0 we for instance get $\langle$ empty, empty, complete, empty $\rangle \in \operatorname{traces}($ Basket 0$),\langle$ empty, empty, empty, ... $\rangle \in$ infinites $($ Basket 0$)$ and divergences $($ Basket 0$)=\{\}$. The infinite sequence of predicates $P_{i}$ for deriving infinite traces is here always $P_{i}=$ true for all $i$.

\section{Refinement}

In this paper, we intend to give a CSP account of Event-B refinement. The previous section provides us with a technique for relating Event-B machines to the semantic domain of CSP processes. Next, we will briefly rephrase the refinement concepts in CSP and Event-B before explaining Event-B refinement in terms of CSP refinement.

\subsection{Event-B refinement}

In Event-B, the (intended) refinement relationship between machines is directly written into the machine definitions. As a consequence of writing a refining machine, a number of proof obligations come up. Here, we assume a machine and its refinement take the following form:

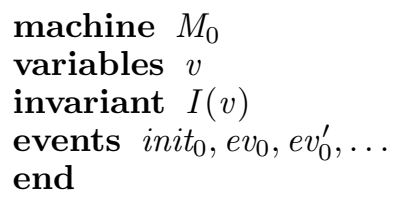

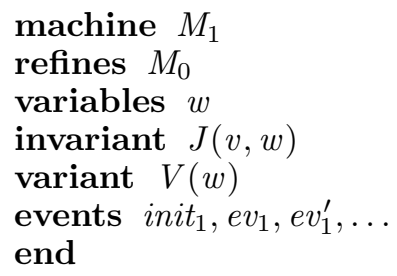

The machine $M_{0}$ is actually refined by machine $M_{1}$, written $M_{0} \preccurlyeq M_{1}$, if the given linking invariant $J$ on the variables of the two machines is established by their initialisations, and preserved by all events. Any transition performed by a concrete event of $M_{1}$ can be matched by a step of the corresponding abstract event of $M_{0}$ (or skip for newly introduced events) to maintain $J$. This is similar to the approach of downwards simulation data refinement [DB01]. We next look at this in more detail, and give the proof obligations associated with these conditions. Formally, the refinement relation $M_{0} \preccurlyeq M_{1}$ between abstract machine $M_{0}$ and concrete machine $M_{1}$ holds if the four proof obligations given below all hold: FIS_REF, GRD_REF, INV_REF and WFD_REF. The first three must hold for all events, and the fourth gives the additional requirement for convergent and anticipated events.

First of all, we need to look at events again. Figure 3 gives the shape of an event and a refinement 


\begin{tabular}{|cc|}
\hline$e v_{0} \hat{=}$ & $e v_{1} \widehat{=}$ \\
status $s t_{0}$ & refines $e v_{0}$ \\
when & status $s t_{1}$ \\
$G(v)$ & when \\
then & $H(w)$ \\
$v: \mid B A 0\left(v, v^{\prime}\right)$ & then \\
end & $w: \mid B A 1\left(w, w^{\prime}\right)$ \\
& end
\end{tabular}

Fig. 3. An event and a refinement of it

\begin{tabular}{|l}
\hline$e v_{1} \widehat{=}$ \\
status $s t_{1}$ \\
when \\
$\quad H(w)$ \\
then \\
end $w: \mid B A 1\left(w, w^{\prime}\right)$ \\
\end{tabular}

Fig. 4. A new event not refining any event

of it. Fig. 4 gives the shape of a new event, which (implicitly) refines skip. We see that an event in the refinement now also gets a status. The status can be ordinary (not noted in this case), or anticipated or convergent. There are different strategies on how these types of events are systematically used in an Event-B development via refinement. The basic idea however is that convergent events are those which are considered internal and must not be executed forever, and anticipated events are those which will take on their roles in later refinement steps only and in these might become convergent. Later, we present two development strategies which precisely fix the use of convergent and anticipated events: Section 6 will give rules for the use of convergent and anticipated events in strategy I, and Section 7 will do so for strategy II.

Both convergent and anticipated events introduce further proof obligations: to prevent execution "forever" the refining machine has to give a variant $V$ (see above in $M_{1}$ ), and $V$ has to be decreased by every convergent event and must not be increased by anticipated events. Before going into the proof obligations in detail, we extend the example of the last section by another machine Basket 1 refining Basket 0 .

The machine Basket1, given in Figure 5, introduces one new possible state for the basket, state_changing. This describes the state of the basket during shopping when the customer inserts or removes goods into / from the basket. A corresponding event change brings the basket into this state. This event has status anticipated: we have not yet decided on its real use. Besides defining the possible values for state, the invariant now also specifies the link between the variables of Basket 0 and Basket1, and we see that the new state state_changing matches both of the old states.

To see that Basket 1 is indeed a refinement of Basket 0 we next describe each of the proof obligations in turn. We have simplified them from their form in [MAV05] by removing explicit references to sets and constants. Alternative forms of these proof obligations are given in [Abr10, Section 5.2: Proof Obligation Rules].

FIS_REF: Feasibility Feasibility of an event is the property that, if the event is enabled (i.e., the guard is true), then there is some after-state. In other words, the body of the event will not block when the event is enabled.

The rule for feasibility of a concrete event is:

\begin{tabular}{|l|l|}
\hline & $I(v) \wedge J(v, w) \wedge H(w)$ \\
$\exists w^{\prime} . B A 1\left(w, w^{\prime}\right)$ & FIS_REF \\
\hline
\end{tabular}




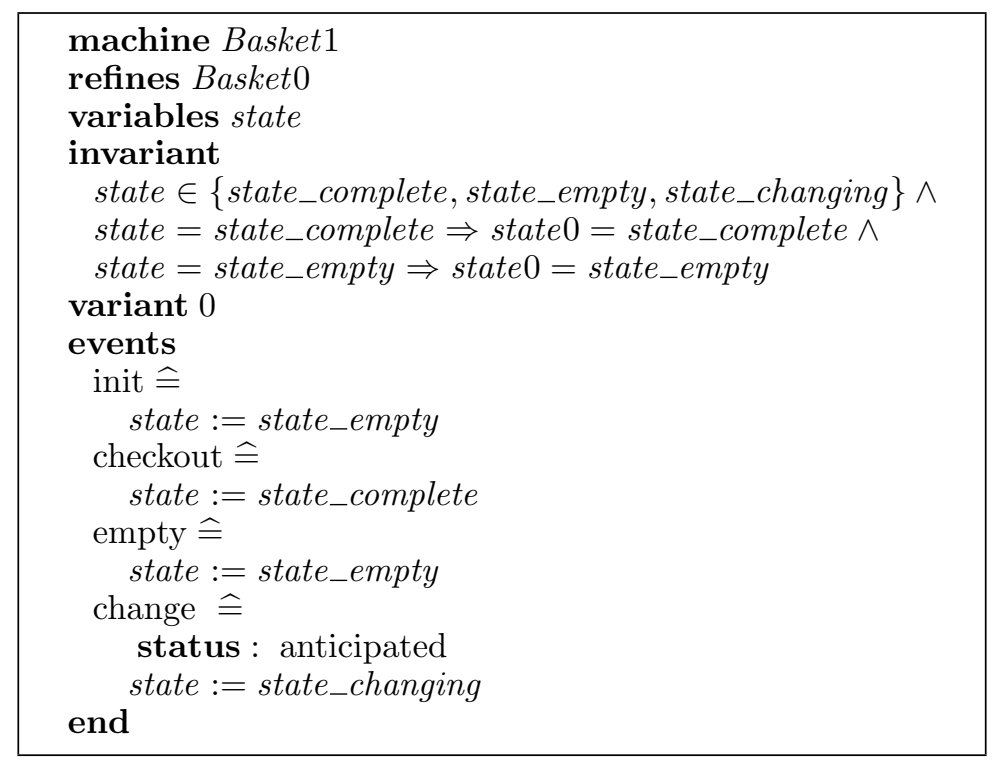

Fig. 5. Event-B machine Basket1

The proof obligation FIS_REF is easy to show for all the events of Basket1 as all events have simple assignments instead of before-after-predicates.

GRD_REF: Guard Strengthening This requires that when a concrete event is enabled, then so is the abstract one. The rule is:

$$
\begin{array}{|l|l|}
\qquad \begin{array}{l}
I(v) \wedge J(v, w) \wedge H(w) \\
G(v)
\end{array} & \text { GRD_REF } \\
\hline
\end{array}
$$

Showing GRD_REF for Basket1 is straightforward as well as both abstract and concrete events have guards equal to true. Later, we will see more complicated refinements. Observe that this proof obligation is trivial if the abstract event is skip, since in that case $G(v)$ is true.

INV_REF: Simulation This ensures that the occurrence of events in the concrete machine can be matched in the abstract one (including the initialization event). If there is an abstract event then the rule is:

\begin{tabular}{|l|l|}
\hline & $I(v) \wedge J(v, w) \wedge H(w) \wedge B A 1\left(w, w^{\prime}\right)$ \\
$\exists v^{\prime} .\left(B A 0\left(v, v^{\prime}\right) \wedge J\left(v^{\prime}, w^{\prime}\right)\right)$ & INV_REF \\
\hline
\end{tabular}

New events are treated as refinements of skip. In this case the abstract state does not change (i.e., BA0 is the identity relation), and the rule is

$$
\begin{array}{l|l}
\vdash(v) \wedge J(v, w) \wedge H(w) \wedge B A 1\left(w, w^{\prime}\right) & \text { INV_REF } \\
\left.J\left(v, w^{\prime}\right)\right) & \text { INE }
\end{array}
$$

For Basket1, event change is a new event. It corresponds to skip as it is always enabled and only changes the state into state_changing, which corresponds to both of the abstract states state_empty and state_complete. 
The two parts of the variant rule WFD_REF below must hold respectively for all convergent and anticipated events.

WFD_REF: Variant This rule ensures that the proposed variant $V$ satisfies the appropriate properties: that it is a natural number, that it decreases on occurrence of any convergent event, and that it does not increase on occurrence of any anticipated event:

\begin{tabular}{|c|l|}
\hline $\begin{array}{l}I(v) \wedge J(v, w) \wedge H(w) \wedge B A 1\left(w, w^{\prime}\right) \\
V(w) \in \mathbb{N} \wedge V\left(w^{\prime}\right)<V(w)\end{array}$ & $\begin{array}{l}\text { WFD_REF } \\
\text { (convergent event) }\end{array}$ \\
\hline $\begin{array}{l}I(v) \wedge J(v, w) \wedge H(w) \wedge B A 1\left(w, w^{\prime}\right) \\
V(w) \in \mathbb{N} \wedge V\left(w^{\prime}\right) \leqslant V(w)\end{array}$ & $\begin{array}{l}\text { WFD_REF } \\
\text { (anticipated event) }\end{array}$ \\
\hline
\end{tabular}

The variant used for Basket 0 is a constant and thus kept by the anticipated event change. This completes the proof that Basket 1 is a refinement of Basket0.

Event-B also allows a variety of further optional proof obligations on refinement steps, depending on what is appropriate for the application. These capture additional properties concerned with liveness (enabledness of sets of events) and deadlock-freedom (enabledness of at least one event), and are not the concern of this paper.

\subsection{CSP refinement}

Based on the semantic domains of traces, failures, divergences and infinite traces, different forms of refinement can be given for CSP. The basic idea underlying these concepts is, however, always the same: the refining process should not exhibit a behaviour which was not possible in the refined process. The different semantic domains then supply us with different forms of "behaviour". In this paper we will use the following refinement relation, based on traces and divergences:

$$
\begin{aligned}
P \sqsubseteq T D I Q \widehat{=} & \operatorname{traces}(Q) \subseteq \operatorname{traces}(P) \\
& \wedge \operatorname{divergences}(Q) \subseteq \operatorname{divergences}(P) \\
& \wedge \text { infinites }(Q) \subseteq \text { infinites }(P)
\end{aligned}
$$

Refinement in Event-B also allows for the possibility of introducing new events. CSP refinement as defined above, however, assumes the alphabet of the processes to be the same. As our objective is to formally state the relationship between Event-B machines in a refinement chain via a form of CSP refinement, we need a way of incorporating additional events into process refinement. As a first idea, we could hide the new events in the refining process, and only afterwards check for refinement. Hiding potentially introduces divergences, namely, when there is an infinite sequence of new events in the infinite traces. When this is the case, we have no $\sqsubseteq_{T D I}$ relationship. Thus hiding gives us the appropriate treatment of new events only in the case where no infinite sequences of new events occur (and this is what Event-B proof obligations guarantee for some sort of new events). As an alternative way of treating new events, we will use $P\|\| R U N_{N}$ as a lazy abstraction operator [Ros98]. $R U N_{N}$ defines a divergence-free process capable of executing any order of events from the set $N$ :

$$
\begin{aligned}
& \text { divergences }\left(R U N_{N}\right)=\{\} \\
& \text { traces }\left(R U N_{N}\right)=N^{*} \\
& \text { infinites }\left(R U N_{N}\right)=N^{\omega}
\end{aligned}
$$

This process together with the interleaving operator will enable us to characterise Event-B refinement introducing new events in CSP terms. The $R U N_{N}$ process gives no restriction on the behaviour of new events. Thus comparing Event-B machines $P$ (or better, its CSP semantics) and $P^{\prime}$ which has introduced new events $N$ via checking of $P\|\| R U N_{N} \sqsubseteq T D I P^{\prime}$ would mean that we check whether there is a refinement relation concerning the "old" events while allowing for every behaviour of the new events. 
The following lemma gives the relationship between refinement involving interleaving, and refinement involving hiding.

Lemma 4.1. If $P_{0} \||| R U N_{N} \sqsubseteq_{T D I} P_{1}$ and $N \cap \alpha P_{0}=\{\}$ and $P_{1} \backslash N$ is divergence-free, then $P_{0} \sqsubseteq_{T D I} P_{1} \backslash$ $N$.

Proof: Assume that (1) $P_{0}\|\| R U N_{N} \sqsubseteq T D I P_{1},(2) N \cap \alpha P_{0}=\{\}$ and (3) $P_{1} \backslash N$ is divergence-free. We need to show that the (finite and infinite) traces as well as divergences of $P_{1} \backslash N$ are contained in those of $P_{0}$.

Traces Let $t r \in \operatorname{traces}\left(P_{1} \backslash N\right)$. By semantics of hiding there is some $t r^{\prime} \in \operatorname{traces}\left(P_{1}\right)$ s.t. $t r^{\prime} \backslash N=t r$. By (1) $t r^{\prime} \in \operatorname{traces}\left(P_{0}|| \mid R U N_{N}\right)$. By (2) and the semantics of $\| \mid$ we get $t r^{\prime} \backslash N \in \operatorname{traces}\left(P_{0}\right)$ and thus $\operatorname{tr} \in \operatorname{traces}\left(P_{0}\right)$.

Divergences By (3) divergences $\left(P_{1} \backslash N\right)=\{\}$, thus nothing to be proven here.

Infinites Let $u \in$ infinites $\left(P_{1} \backslash N\right)$. By the semantics of hiding there is some $u^{\prime} \in$ infinites $\left(P_{1}\right)$ such that $u^{\prime} \backslash N=u$ and $\#\left(u^{\prime} \backslash N\right)=\infty$. By (1) $u^{\prime} \in \operatorname{infinites}\left(P_{0} \| R U N_{N}\right)$ and by (2) and semantics of interleave we get $u^{\prime} \backslash N=u \in \operatorname{infinites}\left(P_{0}\right)$.

As a side remark: The reverse direction holds as well. Note that we do not need the condition on alphabets here (as $N \cap \alpha P_{0}=\{\}$ follows from $\left.P_{0} \sqsubseteq_{T D I} P_{1} \backslash N\right)$.

Lemma 4.2. If $P_{0} \sqsubseteq_{T D I} P_{1} \backslash N$ and $P_{1} \backslash N$ divergence-free, then $P_{0} \| \mid R U N_{N} \sqsubseteq T D I P_{1}$.

Proof: Assume that (1) $P_{0} \sqsubseteq T D I P_{1} \backslash N$ and (2) $P_{1} \backslash N$ divergence-free.

Traces Let $t r \in \operatorname{traces}\left(P_{1}\right)$. Then by semantics of hiding $t r \backslash N \in \operatorname{traces}\left(P_{1} \backslash N\right)$, and thus by (1) $\operatorname{tr} \backslash N \in \operatorname{traces}\left(P_{0}\right)$. Furthermore $\operatorname{tr}\left\lceil N \in \operatorname{traces}\left(R U N_{N}\right)\right.$, and $\operatorname{tr} \in(\operatorname{tr} \backslash N\|\| \operatorname{tr} \backslash N)$. Thus $\operatorname{tr} \in \operatorname{traces}\left(P_{0} \| \mid R U N_{N}\right)$.

Divergence As $P_{1} \backslash N$ is divergence-free, so is $P_{1}$, hence nothing to be shown here.

Infinites Let $u \in \operatorname{infinites}\left(P_{1}\right)$. As $P_{1} \backslash N$ is divergence-free, $\#(u \backslash N)=\infty$. Thus by (1) $u \backslash N \in$ infinites $\left(P_{0}\right)$. The projection onto $N, u \uparrow N$ is either finite or infinite, and $u \uparrow N \in \operatorname{traces}\left(R U N_{N}\right)$ or $u \uparrow N \in$ infinites $\left(R U N_{N}\right)$. In both cases $u \in(u \backslash N \| \mid u \uparrow N)$ and thus $u \in$ infinites $\left(P_{0} \|\right.$ RUN $\left._{N}\right)$.

Both an interleaving with the $R U N_{N}$ process and hiding of $N$ can be used as a way of abstracting away from particular new events $N$ occurring in a process $P_{1}$ but not in $P_{0}$. The two lemmas show that in the absence of a divergence on $N$ in $P_{1}$ (i.e., $P_{1}$ has no infinite sequence with events eventually only from $N$ ) these two can be considered as dual.

\section{Event-B refinement as CSP refinement}

With these definitions in place, we can now look at our main issue, the characterisation of Event-B refinement via CSP refinement. Here, we in particular need to look at the different forms of events in Event-B during refinement. Events can have status convergent or anticipated, or might have no status. This partitions the set of events of $M$ into three sets: anticipated $A$, convergent $C$, and the remaining ones, ordinary events $O$ (neither anticipated nor convergent). The alphabet of $M$, the set of all possible events, is thus given by $\alpha M=A \cup C \cup O$. In the CSP refinement, these will take different roles.

A system development in Event-B via refinement usually proceeds in several steps, gradually introducing more detail into the specification, i.e., we will have a sequence of machines $M_{0}, M_{1}, \ldots, M_{n}$ related by refinement. Now consider an Event-B Machine $M_{i}$ and its refinement $M_{i+1}: M_{i} \preccurlyeq M_{i+1}$. The machine $M_{i}$ has anticipated events $A_{i}$, convergent events $C_{i}$, and ordinary events $O_{i}$, and $M_{i+1}$ similarly has event sets $A_{i+1}, C_{i+1}$, and $O_{i+1}$. Each event $e v_{i}$ in $M_{i}$ is refined by at least one event of $M_{i+1}$ (if no such event appears in $M_{i+1}$ we assume the event to be refined by itself). Each event $e v_{i+1}$ in $M_{i+1}$ either refines a single event $e v_{i}$ in $M_{i}$ (indicated by the clause 'refines $e v_{i}$ ' in the description of $e v_{i+1}$ ) or does not refine any event of $M_{i}$. The set of new events $N_{i+1}$ comprises those events which are not refinements of events in $M_{i} . M_{i} \preccurlyeq M_{i+1}$ thus induces a partial surjective function $f_{i+1}: \alpha M_{i+1} \nrightarrow \alpha M_{i}$ where $f_{i+1}\left(e v_{i+1}\right)=e v_{i} \Leftrightarrow e v_{i+1}$ refines $e v_{i}$. Observe that $\alpha M_{i+1}$ is partitioned by $f_{i+1}^{-1}\left(\alpha M_{i}\right)$ and $N_{i+1}$. 
For the two Event-B machines Basket 0 and Basket 1 we have $O_{0}=\{$ empty, checkout $\}, A_{0}=C_{0}=\{\}$, and $O_{1}=\{$ empty, checkout $\}$ and $N_{1}=A_{1}=$ \{change $\}$. The function $f_{1}$ thus is the mapping $\{$ empty $\mapsto$ empty, checkout $\mapsto$ checkout $\}$.

\subsection{New events}

The above definitions fix the relation between events in $M_{i}$ and $M_{i+1}$ independent of a particular development strategy. With these at hand, we can look at the CSP semantics of Event-B and see what the corresponding notion of refinement in CSP is. First of all, for the new events arising in the refinement we can use the lazy abstraction operator via the $R U N$ process to get our desired result, disregarding the issue of divergence for a moment.

The following lemma gives our first result on the relationship between Event-B refinement and CSP refinement. Observe that we use priming notation when considering the general relationship between a machine $M$ and its refinement $M^{\prime}$, rather than the indexing notation used for refinement chains.

Lemma 5.1. If $M \preccurlyeq M^{\prime}$ and the refinement introduces new events $N^{\prime}$ and uses the mapping $f^{\prime}$, then $f^{\prime-1}(M)\|\| R U N_{N^{\prime}} \sqsubseteq_{T D I} M^{\prime}$.

Proof: We assume state variables of $M$ and $M^{\prime}$ named as given above, i.e., state variables of $M$ are $v$ and of $M^{\prime}$ are $w$. Let $\operatorname{tr}=\left\langle a_{1}, \ldots, a_{n}\right\rangle \in \operatorname{traces}\left(M^{\prime}\right)$. We need to show that $\operatorname{tr} \in \operatorname{traces}\left(f^{\prime-1}(M) \| \operatorname{RUN}_{N^{\prime}}\right)$. First of all note that the interleaving operator merges the traces of two processes together, i.e., the traces of $f^{\prime-1}(M) \mid \| R U N_{N^{\prime}}$ are simply those of $f^{\prime-1}(M)$ with new events arbitrarily inserted. The proof proceeds by induction on the length of the trace.

Induction base Assume $n=0$, i.e., $\operatorname{tr}=\langle\rangle$. This case is trivial, since \langle\rangle$\in \operatorname{traces}\left(f^{\prime-1}(M) \| R U N_{N^{\prime}}\right)$.

Induction step Assume that for a trace $t_{0}=\left\langle a_{1}, \ldots, a_{j-1}\right\rangle \in \operatorname{traces}\left(M^{\prime}\right)$ we have already shown that $\operatorname{tr}_{0} \in \operatorname{traces}\left(f^{\prime-1}(M) \mid \| R U N_{N^{\prime}}\right)$ and this has led us to a pair of states $v_{j-1}, w_{j-1}$ such that $J\left(v_{j-1}, w_{j-1}\right)$. (Observe that in the case where $t r_{0}=\langle\rangle$ the initialisation event $i n i t^{\prime}$ has been executed bringing the machine $M^{\prime}$ into a state $w_{0}$. By INV_REF on init, we find a state $v_{0}$ such that $J\left(v_{0}, w_{0}\right)$.)

Now two cases need to be considered:

1. $a_{j} \notin N^{\prime}$ : Assume $a_{j}$ in $M^{\prime}$ to be of the form

when $H(w)$ then $w: \mid B A^{\prime}\left(w, w^{\prime}\right)$ end

and $f^{\prime}\left(a_{j}\right)$ in $M$ of the form

when $G(v)$ then $v: \mid B A\left(v, v^{\prime}\right)$ end

Since $a_{j}$ is executed in $w_{j-1}$ we have $H\left(w_{j-1}\right)$. By GRD_REF we thus get $G\left(v_{j-1}\right)$. Furthermore, for $w_{j}$ with $B A^{\prime}\left(w_{j-1}, w_{j}\right)$ we find, by INV_REF, a state $v_{j}$ such that $J\left(v_{j}, w_{j}\right)$ and $B A\left(v_{j-1}, v_{j}\right)$. Hence $t r_{0} \frown\left\langle a_{j}\right\rangle \in \operatorname{traces}\left(f^{\prime-1}(M) \| R U N_{N^{\prime}}\right)$.

2. $a_{j} \in N^{\prime}$ : Similar to the previous case. Here, $a_{j}$ refines skip and thus $v_{j}=v_{j-1}$ and the event $a_{j}$ comes from $R U N_{N^{\prime}}$.

In the same way we can carry out a proof for infinite traces. For divergences it is trivial since divergences $\left(M^{\prime}\right)=$ \{\} .

For our example, we thus get $f_{1}^{-1}$ (Basket 0$)\|\| R U N_{\{\text {change }\}} \sqsubseteq T D I$ Basket 1 .

This lemma can be generalised to a chain of refinement steps. For this, we assume that we are given a sequence of Event-B machines $M_{i}$ with their associated processes $P_{i}$, and every refinement step introduces some set of new events $N_{i}$.

Theorem 5.2. If a sequence of processes $P_{i}$, mappings $f_{i}$, and sets $N_{i}$ are such that

$$
f_{i+1}^{-1}\left(P_{i}\right) \| R U N_{N_{i+1}} \sqsubseteq_{T D I} P_{i+1}
$$

for each $i$, then

$$
f_{n}^{-1}\left(\ldots\left(f_{1}^{-1}\left(P_{0}\right)\right) \ldots\right)\|\| R U N_{f_{n}^{-1}\left(\ldots f_{2}^{-1}\left(N_{1}\right) \ldots\right) \cup \ldots \cup f_{n}^{-1}\left(N_{n-1}\right) \cup N_{n}} \sqsubseteq_{T D I} P_{n}
$$




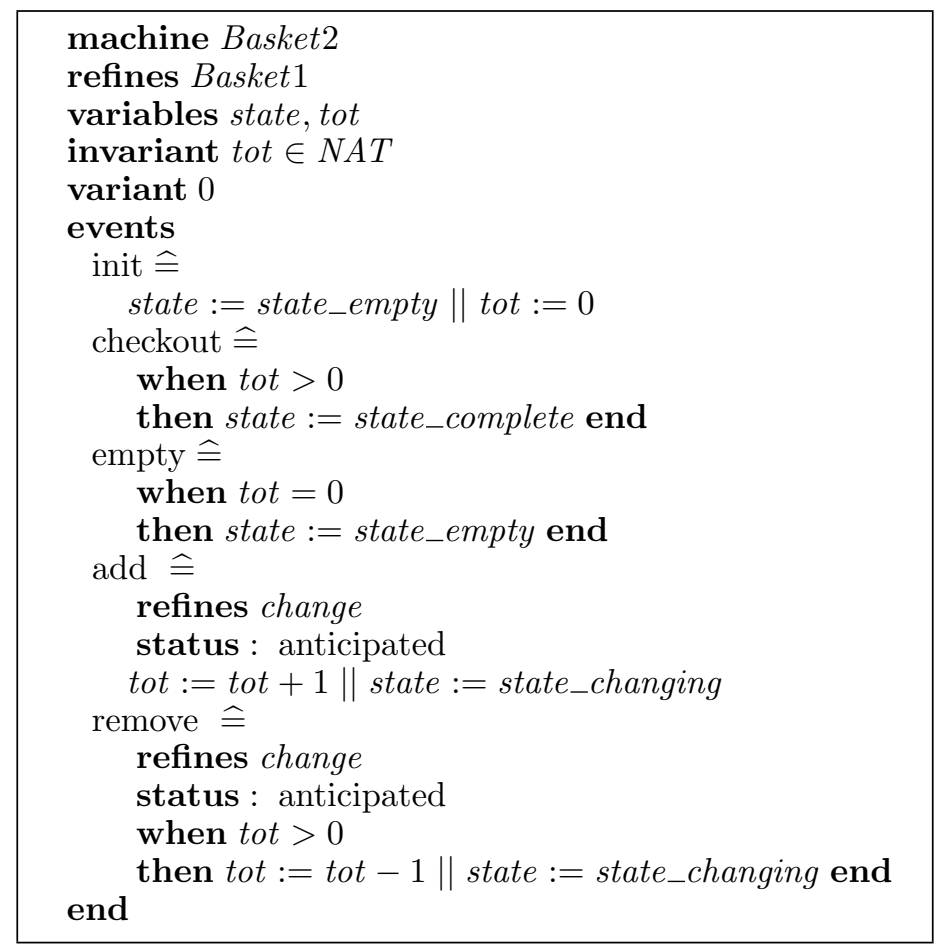

Fig. 6. Event-B machine Basket2

Proof: Two successive refinement steps combine to provide a relationship between $P$ and $P^{\prime \prime}$ of the same form as Line 1 above, as follows:

$$
\begin{aligned}
& f^{\prime \prime-1}\left(P^{\prime}\right)\|\| R U N_{N^{\prime \prime}} \quad \sqsubseteq_{T D I} \quad P^{\prime \prime} \quad \text { (given) } \\
& f^{\prime \prime-1}\left(f^{\prime-1}(P)\|\| R U N_{N^{\prime}}\right) \| R U N_{N^{\prime \prime}} \sqsubseteq_{T D I} \quad P^{\prime \prime} \quad \text { (line }(1) \text {, monotonicity of } f^{\prime \prime-1} \text {, transitivity of } \sqsubseteq \text { ) } \\
& f^{\prime \prime-1}\left(f^{\prime-1}(P)\right)\|\| R U N_{f^{\prime \prime-1}\left(N^{\prime}\right)} \mid \| R U N_{N^{\prime \prime}} \sqsubseteq_{T D I} \quad P^{\prime \prime} \quad\left(\text { Law: } f^{-1}(P\|\mid\| Q)=f^{-1}(P)\|\| f^{-1}(Q)\right) \\
& f^{\prime \prime-1}\left(f^{\prime-1}(P)\right) \mid \| R U N_{f^{\prime \prime}-1}\left(N^{\prime}\right) \cup N^{\prime \prime} \quad \sqsubseteq_{T D I} \quad P^{\prime \prime} \quad\left(\text { Law: } R U N_{A} \| R U N_{B}=R U N_{A \cup B}\right)
\end{aligned}
$$

Hence the whole chain of refinement steps can be collected together, yielding the result.

To see how this works for our example, we extend our chain of refinements by another machine, Basket2, shown in Figure 6. In Basket 2 we split one event, namely change, into two, adding and removing goods from the basket. Both these events are tagged as anticipated. For this refinement we thus have $N_{2}=\{\}$, $A_{2}=\{$ add, remove $\}, C_{2}=\{\}$ and $f_{2}:\{a d d \mapsto$ change, remove $\mapsto$ change $\}$.

The above lemma on refinement chains and new events then gives us the following result, relating the initial machine Basket 0 and Basket2.

$$
f_{2}^{-1}\left(f_{1}^{-1}(\text { Basket } 0)\right)|| \mid R U N_{\{a d d, \text { remove }\}} \sqsubseteq_{T D I} \text { Basket } 2 \text {. }
$$

This result states that traces, infinite traces, and divergences of Basket 2 must also be possible for $f_{2}^{-1}\left(f_{1}^{-1}(\right.$ Basket 0$\left.)\right)\|\| R U N_{\{a d d, \text { remove }\}}$. For example, consider the trace

$\langle$ add, add, checkout, remove, remove, empty $\rangle \in \operatorname{traces}($ Basket 2$)$

We find that

$$
\langle\text { checkout, empty }\rangle \in \operatorname{traces}\left(f_{2}^{-1}\left(f_{1}^{-1}(\text { Basket } 0)\right)\right)
$$

and

$$
\langle a d d, \text { add, remove, remove }\rangle \in \operatorname{traces}\left(R U N_{\{a d d, \text { remove }\}}\right)
$$


so indeed we find that

$\langle a d d$, add, checkout, remove, remove, empty $\rangle \in \operatorname{traces}\left(f_{2}^{-1}\left(f_{1}^{-1}(\right.\right.$ Basket 0$\left.\left.)\right) \| \mid R U N_{\{\text {add,remove }\}}\right)$

The infinite trace $\langle a d d\rangle^{\omega}$ infinites(Basket2), and it is also in infinites $\left(R U N_{\{a d d, \text { remove }\}}\right)$, hence in infinites $\left(f_{2}^{-1}\left(f_{1}^{-1}(\right.\right.$ Basket 0$\left.\left.)\right)\|\| R U N_{\{\text {add,remove }\}}\right)$.

There are no divergences at any refinement level: no divergences can be introduced through refinement.

\subsection{Convergent and anticipated events}

The previous result lets us relate the first and last Event-B machine in a chain of refinements. Due to the lazy abstraction operator (and the resulting possibility of defining refinement without hiding new events), we considered divergence-free processes there: all processes $P_{i}$ representing Event-B machines, are divergence free by definition. However, Event-B refinement is concerned with a particular form of divergence and its avoidance. A sort of divergence would arise when new events (or more specifically, convergent events) could be executed forever, and this is what the proof obligations for variants rule out.

We would like to capture the impact of convergence and anticipated sets of events in the CSP semantics as well. To do so, we first of all define the specification predicate

$$
C A(C, O)(u) \hat{=}(\#(u\lceil C)=\infty \Rightarrow \#(u \uparrow O)=\infty)
$$

Intuitively, this states that all infinite traces having infinitely many convergent $(C)$ events also have infinitely many $(O)$ ordinary events (and thus cannot execute convergent events alone forever). In this case we say that the Event-B machine does not diverge on $C$ events.

Definition 5.3. Let $M$ be an Event-B machine with its alphabet $\alpha M$ containing event sets $C$ and $O$ with $C \cap O=\{\} . M$ does not diverge on $C$ events if $M$ sat $C A(C, O)$.

Observe that if a machine has no convergent events $(C=\{\})$ then it trivially satisfies the specification $C A(C, O)$. Note that the first machine $M_{0}$ in an Event-B refinement chain has no convergent events convergent events only come into play during refinement - so it will satisfy $C A(C, O)$.

Lemma 5.4. If $M \preccurlyeq M^{\prime}$, and $M^{\prime}$ has convergent, anticipated, and ordinary sets of events $C^{\prime}, A^{\prime}$, and $O^{\prime}$ respectively, then $M^{\prime}$ sat $C A\left(C^{\prime}, O^{\prime}\right)$.

Proof: We prove this by contradiction. Assume $\neg M^{\prime}$ sat $C A\left(C^{\prime}, O^{\prime}\right)$. Then there is some $u \in$ infinites $\left(M^{\prime}\right)$ such that $\#\left(u\left\lceil C^{\prime}\right)=\infty\right.$ and $\#\left(u\left\lceil O^{\prime}\right)<\infty\right.$. Then there must be some $t r_{0}, u^{\prime}$ such that $u=t r_{0} \frown u^{\prime}$ with $u^{\prime} \in\left(C^{\prime} \cup A^{\prime}\right)^{\omega}$ (i.e., $t r_{0}$ is a prefix of $u$ containing all the $O^{\prime}$ events). Moreover, $\# u^{\prime} \uparrow C^{\prime}=\infty$.

Now since $M \preccurlyeq M^{\prime}$ we have by GRD_REF and INV_REF that there is some pair of states $(v, w)$ (abstract and concrete state) reached after executing $t r_{0}$ for which $J(v, w)$ and $I(v)$ is true. Furthermore, $V(w)$ is a natural number. Also by $M \preccurlyeq M^{\prime}$ we have an infinite sequence of pairs of states $\left(v_{i}, w_{i}\right)$ (for the remaining infinite trace $\left.u^{\prime}\right)$ such that $J\left(v_{i}, w_{i}\right)$. Since each event in $u^{\prime}$ is in $A^{\prime}$ or $C^{\prime}$ we have from WFD_REF that $V\left(w_{i+1}\right) \leqslant V\left(w_{i}\right)$ for each $i$. Further, for infinitely many $i$ 's (i.e., those events in $C^{\prime}$ ) we have $V\left(w_{i+1}\right)<V\left(w_{i}\right)$. Thus we have a sequence of values $V\left(w_{i}\right)$ decreasing infinitely often without ever increasing. This contradicts the fact that the $V\left(w_{i}\right) \in \mathbb{N}$.

A number of further interesting properties can be deduced for the specification predicate $C A$.

Lemma 5.5. Let $P$ be a CSP process and $C, C^{\prime}, O \subseteq \alpha P$ nonempty finite sets of events.

1. If $P$ sat $C A(C, O)$ then $f^{-1}(P)$ sat $C A\left(f^{-1}(C), f^{-1}(O)\right)$.

2. If $P$ sat $C A(C, O)$ and $N \cap C=\{\}$ then $P\|\| R U N_{N}$ sat $C A(C, O)$.

3. If $P$ sat $C A(C, O)$ and $P$ sat $C A\left(C^{\prime}, C \cup O\right)$ then $P$ sat $C A\left(C \cup C^{\prime}, O\right)$.

4. If $P$ sat $C A(C, O)$ and $C \cap O=\{\}$ then $P \backslash C$ is divergence-free.

5. If $P$ sat $C A(C, O)$ then $P$ sat $C A(C, O \cup X)$ for every set $X$ of events.

Proof: 
1. Assume that $u \in$ infinites $\left(f^{-1}(P)\right)$ and $\#\left(u\left\lceil f^{-1}(C)\right)=\infty\right.$. From the first we get $f(u) \in$ infinites $(P)$. From the latter it follows that $\#(f(u)\lceil C)=\infty$. With $P$ sat $C A(C, O)$ we have $\#(f(u)\lceil O)=\infty$ and hence $\#\left(u\left\lceil f^{-1}(O)\right)=\infty\right.$.

2. Let $u \in \operatorname{infinites}\left(P \| R U N_{N}\right)$ and $\#(u\lceil C)=\infty$. With $N \cap C=\{\}$ we get $\#((u \backslash N) \uparrow C)=\infty$. By definition of || $\mid$ we have $u \backslash N \in$ infinites $(P)(u \backslash N$ is infinite since $\#((u \backslash N) \uparrow C)=\infty)$. By $P$ sat $C A(C, O)$ we get $\#((u \backslash N) \uparrow O)=\infty$, hence $\#(u \uparrow O)=\infty$.

3. Let $u \in \operatorname{infinites}(P)$ such that $\#\left(u \uparrow\left(C \cup C^{\prime}\right)\right)=\infty$. Both $C$ and $C^{\prime}$ are finite sets hence either $\#\left(u\lceil C)=\infty\right.$ or $\#\left(u\left\lceil C^{\prime}\right)=\infty\right.$ (or both). In the first case we get $\#(u\lceil O)=\infty$ by $P$ sat $C A(C, O)$. In the second case it follows that $\#(u\lceil(C \cup O))=\infty$ and hence again $\#(u\lceil C)=\infty$ or directly $\#(u \uparrow O)=\infty$.

4. First of all note that if $P$ sat $C A(C, O)$ then $P$ is divergence free. Now assume that there is a trace $\operatorname{tr} \in$ divergences $(P \backslash C)$. Then there exists a trace $u \in$ infinites $(P)$ such that $\operatorname{tr}=u \backslash C$, and so $\#(u \backslash C)<\infty$. Hence \# $(u\lceil C)=\infty$. However, as $C \cap O=\{\}, \#(u\lceil O) \neq \infty$ which contradicts $P$ sat $C A(C, O)$.

5. Follows from the fact that if $\#(u \uparrow O)=\infty$ then $\#(u \uparrow O \cup X)=\infty$.

The most interesting of these properties is probably the fourth one: it relates the specification predicate to the definition of divergence freedom in CSP. In CSP, a process does not diverge on a set of events $C$ if $P \backslash C$ is divergence-free.

We also obtain a result for the specification predicate $C A$ with respect to the relationship between a machine and a refinement of it.

Lemma 5.6. Let $M \preccurlyeq M^{\prime}$ with an associated refinement function $f^{\prime}$. Let $C, C^{\prime}, O$ and $O^{\prime}$ be convergent and ordinary sets such that $M$ sat $C A(C, O)$ and $M^{\prime}$ sat $C A\left(C^{\prime}, O^{\prime}\right)$, and $O^{\prime}=f^{\prime-1}(C) \cup f^{\prime-1}(O) \cup X$. The set $X$ is the ordinary events in $M^{\prime}$ that do not refine ordinary or convergent events in $M$. Then:

$$
M^{\prime} \text { sat } C A\left(f^{\prime-1}(C) \cup C^{\prime}, f^{\prime-1}(O) \cup X\right)
$$

Proof: Assume $u \in$ infinites $\left(M^{\prime}\right)$ and $\#\left(u \uparrow\left(f^{\prime-1}(C) \cup C^{\prime}\right)\right)=\infty$. We aim to establish that \# $(u\lceil$ $\left.f^{\prime-1}(O) \cup X\right)=\infty$. We have $\#\left(u\left\lceil f^{\prime-1}(C)\right)=\infty\right.$ or $\#\left(u\left\lceil C^{\prime}\right)=\infty\right.$.

In the former case, Lemma 5.1 yields that $f^{\prime}\left(u\left\lceil f^{-1}(\alpha M)\right) \in \operatorname{infinites}(M)\right.$. Then

$$
\begin{array}{rlrl}
\#\left(u \uparrow f^{\prime-1}(C)\right)=\infty & & \text { (given) } \\
\#\left(f^{\prime}\left(u \uparrow f^{\prime-1}(C)\right) \uparrow C\right)=\infty & \text { (since renaming preserves length) } \\
\#\left(f^{\prime}\left(u\left\lceil f^{\prime-1}(\alpha M)\right) \uparrow C\right)=\infty\right. & \text { (since } C \subseteq \alpha M) \\
\#\left(f^{\prime}\left(u\left\lceil f^{\prime-1}(\alpha M)\right) \uparrow O\right)=\infty\right. & \text { (by } M \text { sat } C A(C, O)) \\
\#\left(u \left\lceilf^{\prime-1}(\alpha M) \uparrow f^{\prime-1}(O)=\infty\right.\right. & \text { (since renaming preserves length) } \\
\#\left(u\left\lceil f^{\prime-1}(O)\right)=\infty\right. & \text { (since } O \subseteq \alpha M) \\
\#\left(u\left\lceil f^{\prime-1}(O) \cup X\right)=\infty\right. & \text { (since } \left.f^{\prime-1}(O) \subseteq f^{\prime-1}(O) \cup X\right)
\end{array}
$$

In the latter case Lemma 5.4 yields that $\#\left(u \uparrow O^{\prime}\right)=\infty$. Then

$$
\begin{aligned}
\#\left(u \uparrow O^{\prime}\right) & =\infty \\
\#\left(u\left\lceil f^{\prime-1}(C) \cup f^{\prime-1}(O) \cup X\right)\right. & =\infty \\
\#\left(u \uparrow f^{\prime-1}(O) \cup X\right)=\infty \vee \#\left(u \uparrow f^{\prime-1}(C)\right) & =\infty
\end{aligned} \quad\left(\text { since } O^{\prime}=f^{\prime-1}(C \cup O) \cup X\right)
$$

The first disjunct is the desired result, the second is the one already treated above.

We use the $C A$ specification predicate to reason about (the absence of) divergence on particular events in an Event-B machine. However, from now on our results will be specific to a particular development strategy, and the way it imposes rules on convergent and anticipated events. Therefore, we next present our first development strategy.

\section{Strategy I}

In an Event-B machine the status of an event fixes its type and thus the proof obligations derived for the event. However, it is not only the status and its proof obligations which influences the corresponding notion 
of refinement in CSP. Another decisive issue is the development strategy. A development strategy essentially fixes how anticipated, convergent and ordinary events are used in a development via refinement, in particular, how the status of an event may change in a refinement step. Though development strategies have not been discussed explicitly in the Event-B literature so far, let alone formalised, it is clear that Event-B designers actually follow strategies (though not necessary all the same). So, in the following we will (for the first time) define (two) development strategies for Event-B.

The first strategy, which we have already presented in [STW11b], assumes that the initial specification $M_{0}$ already fixes the interface of the system to the environment, and later refinement steps only make the internals of the system more precise. We call this approach refinement with no interface extension. The second approach assumes that the interface can be extended in every refinement step, called refinement with interface extension. The role of anticipated and convergent events in these approaches is the following. One idea behind the Event-B refinement proof obligations is that internal events should not be executed forever, i.e., there should not be an infinite sequence of internal events only so as to enable interaction with the environment via the interface. Therefore we have particular proof obligations (using variants) on anticipated and convergent events. In the non-extensible interface approach, all newly introduced events are internal and thus eventually need to be shown to converge. In the extensible interface approach there is the option of a newly introduced event to become part of the interface and thus not to be shown to be convergent.

In this section we will follow the strategy I (no interface extension), and in particular precisely define what rules this approach imposes on refinement. The next section will look at strategy II. With non-extensible interfaces we have the following restrictions on the event sets in a development $M_{0} \preccurlyeq M_{1} \preccurlyeq \ldots \preccurlyeq M_{n}$.

1. in the initial machine all events are ordinary (and thus fix the interface of the system),

2. each event of $M_{i}$ is refined by at least one event of $M_{i+1}$;

3. each new event in $M_{i}$ is either anticipated or convergent;

4. each event in $M_{i+1}$ which refines an anticipated event of $M_{i}$ is itself either convergent or anticipated;

5. refinements of convergent or ordinary events of $M_{i}$ are ordinary in $M_{i+1}$, i.e., they are not given a status;

6. no anticipated events remain in the final machine.

The conditions imposed by the rules are formalised as follows:

1. $A_{0}=C_{0}=\{\}$;

2. $\operatorname{ran}\left(f_{i+1}\right)=A_{i} \cup C_{i} \cup O_{i}$;

3. $N_{i}=\left(A_{i} \cup C_{i}\right) \backslash \operatorname{dom}\left(f_{i}\right)$;

4. $f_{i+1}^{-1}\left(A_{i}\right) \subseteq A_{i+1} \cup C_{i+1}$;

5. $f_{i+1}^{-1}\left(C_{i} \cup O_{i}\right)=f_{i+1}^{-1}\left(C_{i}\right) \cup f_{i+1}^{-1}\left(O_{i}\right)=O_{i+1}$;

6. $A_{n}=\{\}$.

These relationships between the classes of events are illustrated in Fig. 7.

Note that condition 5 satisfies the condition of Lemma 5.6 (with $X=\{\}$ ), and so that lemma applies to refinement steps in strategy I.

To be able to apply this to our example, we extend it with a new machine Basket3.I (depicted in Figure 8) containing for the first time convergent events. In the second development strategy we will take a different route here and instead make a refinement to machine Basket3.II. Here, the basket is extended with variable tot counting the number of goods in the basket, and a boolean variable scanning as a flag for the start of the scanning procedure at the counter. Event checkout starts the scanning procedure and event scan scans every good in the basket. Event empty can happen when the basket is empty. It stops scanning and thereby enables event add again. Observe that while the basket is not scanning, scan cannot occur before checkout occurs. This ensures that the trace $\langle a d d \text {, remove }\rangle^{\omega}$ cannot occur. Since this trace violates $C A\left(C_{3 . I}, O_{3 . I}\right)$, ruling it out is necessary to ensure that $C A\left(C_{3 . I}, O_{3 . I}\right)$ holds on Basket3.I.

Since Basket $2 \preccurlyeq$ Basket $3 . I$ we have that

$$
f_{3 . I}^{-1} \text { (Basket2) ||| RUN } N_{\{\}} \sqsubseteq_{T D I} \text { Basket } 3 . I
$$

No new event is introduced in Basket3.I, and this is equivalent to the simpler formulation

$$
f_{3 . I}^{-1}(\text { Basket } 2) \sqsubseteq T D I \text { Basket } 3 . I
$$




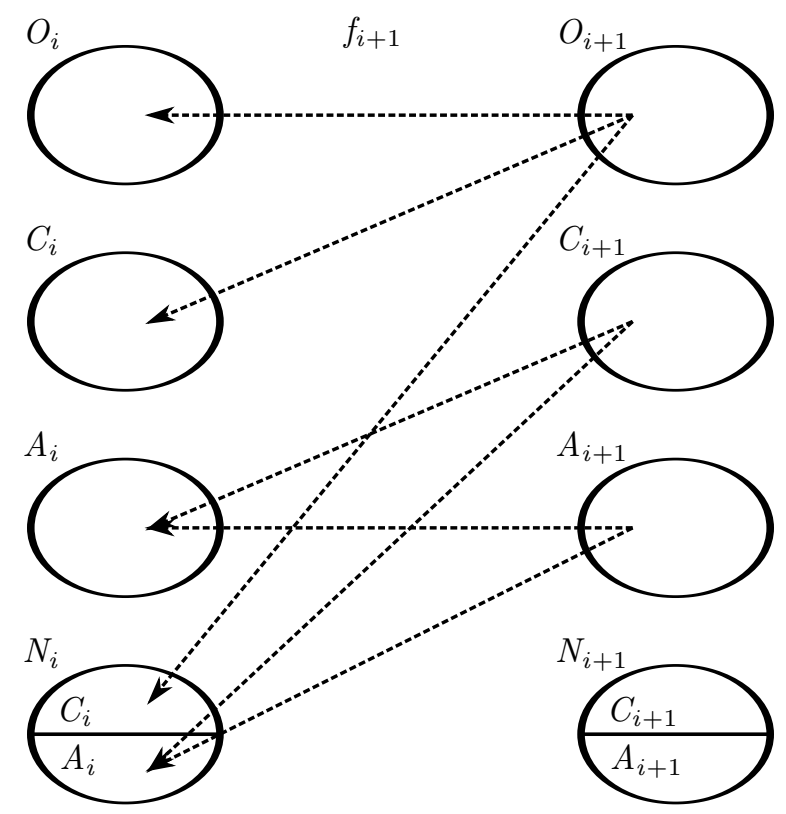

Fig. 7. Relationship between events in a refinement step in the non-extensible interface approach: $f_{i+1}$ maps events in $M_{i+1}$ to events in $M_{i}$ that they refine.

For example, the trace $\langle a d d$, add, checkout, scan, scan, empty $\rangle \in \operatorname{traces}($ Basket3.I) corresponds, via the renaming function $f_{3 . I}$, to the trace $\langle a d d$, add, checkout, remove, remove, empty $\rangle \in \operatorname{traces}($ Basket 2$)$. The results states that any trace of Basket3.I must correspond to some trace of Basket2.

The converse need not hold. The trace $\langle a d d$, remove $\rangle \in \operatorname{traces}($ Basket 2$)$ does not have any associated trace in Basket $3 . I$.

The event scan is tagged convergent and thus the proof obligation WFD_REF needs to be shown. The variant specified in the machine is if scanning = true then tot else 0 . It is decreased by scan as it sets tot to tot -1 . Hence we obtain that Basket $3 . I \backslash\{$ scan $\}$ is divergence-free.

Thus we obtain

We require the following lemma in order to establish Theorem 6.2 subsequently:

Lemma 6.1. If $M_{0} \preccurlyeq M_{1} \preccurlyeq \ldots \preccurlyeq M_{n}$ then

$$
O_{n}=\left(f_{n}^{-1}\left(\ldots f_{1}^{-1}\left(C_{0}\right) \ldots\right) \cup \ldots f_{n}^{-1}\left(C_{n-1}\right)\right) \cup f_{n}^{-1}\left(\ldots f_{1}^{-1}\left(O_{0}\right) \ldots\right)
$$

Proof: By induction on $n$.

Induction base Assume $n=0$. In this case the statement reduces to $O_{0}=O_{0}$ which is trivially true.

Induction step Assume the result for $i$, we aim to establish it for $i+1$. By Condition 5 of strategy I we have that $O_{i+1}=f_{i+1}^{-1}\left(C_{i}\right) \cup f_{i+1}^{-1}\left(O_{i}\right)$. Hence

$$
\begin{aligned}
& O_{i+1}=f_{i+1}^{-1}\left(C_{i}\right) \cup f_{i+1}^{-1}\left(O_{i}\right) \\
& =f_{i+1}^{-1}\left(C_{i}\right) \cup f_{i+1}^{-1}\left(f_{i}^{-1}\left(\ldots f_{1}^{-1}\left(C_{0}\right) \ldots\right) \cup \ldots f_{i}^{-1}\left(C_{i-1}\right)\right) \\
& \cup f_{i}^{-1}\left(\ldots f_{1}^{-1}\left(O_{0}\right) \ldots\right) \quad \text { (inductive hypothesis) } \\
& \left.=\left(f_{i+1}^{-1}\left(\ldots f_{1}^{-1}\left(C_{0}\right) \ldots\right) \cup \ldots f_{i+1}^{-1}\left(C_{i}\right)\right) \cup f_{i+1}^{-1}\left(\ldots f_{1}^{-1}\left(O_{0}\right) \ldots\right)\right) \quad \text { (reordering) }
\end{aligned}
$$

which establishes the case.

The result follows by induction.

We now obtain the following result on refinement chains:

Theorem 6.2. If $M_{0} \preccurlyeq M_{1} \preccurlyeq \ldots \preccurlyeq M_{n}$ then

$$
M_{n} \text { sat } C A\left(\left(f_{n}^{-1}\left(\ldots f_{1}^{-1}\left(C_{0}\right) \ldots\right) \cup \ldots f_{n}^{-1}\left(C_{n-1}\right) \cup C_{n}\right), f_{n}^{-1}\left(\ldots f_{1}^{-1}\left(O_{0}\right) \ldots\right)\right)
$$




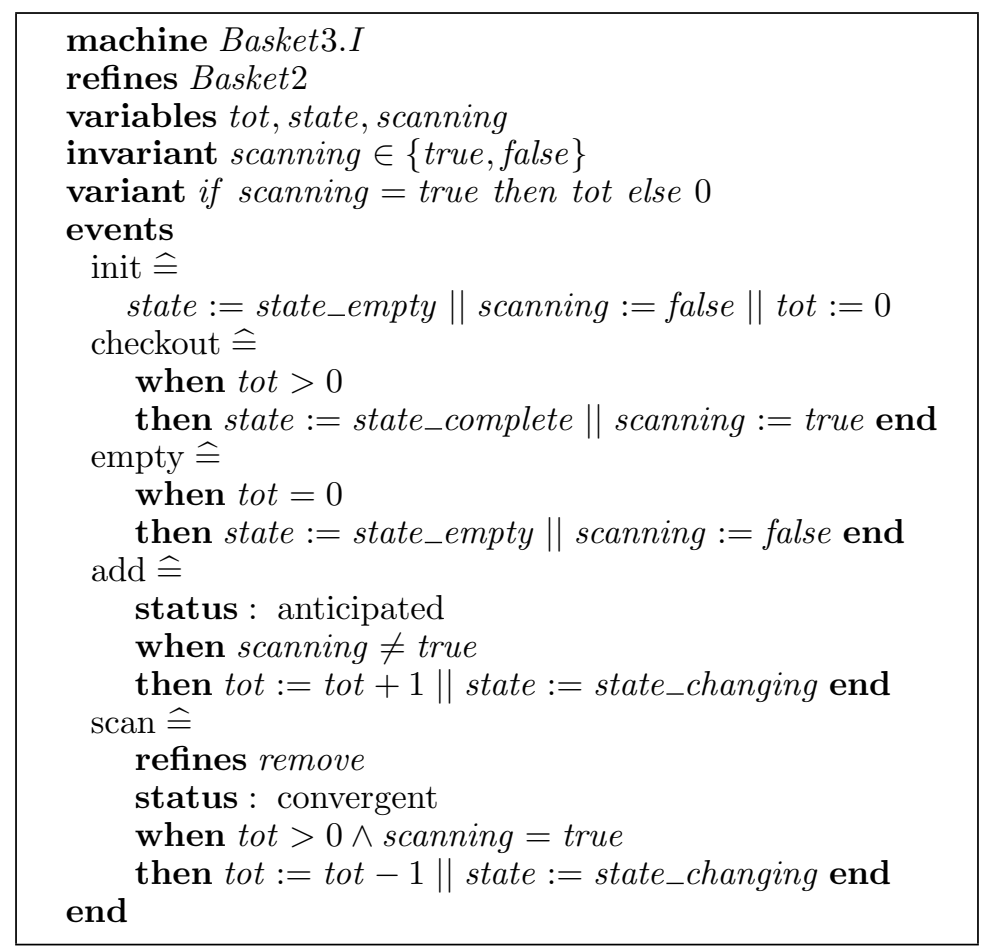

Fig. 8. Event-B machine Basket3.I

Proof: By induction on $n$.

Induction base Assume $n=0$. In this case $C_{0}=\{\}$ since $M_{0}$ contains no convergent events, and so $M_{0}$ sat $C A\left(C_{0}, O_{0}\right)$ is vacuously true. Observe that $C_{0}=\left(f_{n}^{-1}\left(\ldots f_{1}^{-1}\left(C_{0}\right) \ldots\right) \cup \ldots f_{n}^{-1}\left(C_{n-1}\right) \cup C_{n}\right)$, and $\left.O_{0}=f_{n}^{-1}\left(\ldots f_{1}^{-1}\left(O_{0}\right) \ldots\right)\right)$ with $n=0$, establishing the case.

Induction step Assume the result for $i$, we aim to establish it for $i+1$. The inductive hypothesis gives:

$$
M_{i} \text { sat } C A\left(\left(f_{i}^{-1}\left(\ldots f_{1}^{-1}\left(C_{0}\right) \ldots\right) \cup \ldots f_{i}^{-1}\left(C_{i-1}\right) \cup C_{i}\right), f_{i}^{-1}\left(\ldots f_{1}^{-1}\left(O_{0}\right) \ldots\right)\right)
$$

and from Lemma 5.4 we have:

$$
M_{i+1} \text { sat } C A\left(C_{i+1}, O_{i+1}\right)
$$

Now for $M_{i}=M$ and $M_{i+1}=M^{\prime}$ we define

$$
\begin{aligned}
C & =\left(f_{i}^{-1}\left(\ldots f_{1}^{-1}\left(C_{0}\right) \ldots\right) \cup \ldots f_{i}^{-1}\left(C_{i-1}\right) \cup C_{i}\right) \\
O & =f_{i}^{-1}\left(\ldots f_{1}^{-1}\left(O_{0}\right) \ldots\right) \\
C^{\prime} & =C_{i+1} \\
O^{\prime} & =O_{i+1}
\end{aligned}
$$

We observe from Lemma 6.1 that $O^{\prime}=f_{i+1}^{-1}(C) \cup f_{i+1}^{-1}(O)$. It follows from Lemma 5.6 (with $X=\{\}$ ) that

$$
M_{i+1} \text { sat } C A\left(f_{i+1}^{-1}\left(f_{i}^{-1}\left(\ldots f_{1}^{-1}\left(C_{0}\right) \ldots\right) \cup \ldots f_{i}^{-1}\left(C_{i-1}\right) \cup C_{i}\right) \cup C_{i+1}, f_{i+1}^{-1}\left(f_{i}^{-1}\left(\ldots f_{1}^{-1}\left(O_{0}\right) \ldots\right)\right)\right) \text {, }
$$
i.e.,

$$
M_{i+1} \text { sat } C A\left(f_{i+1}^{-1}\left(\ldots f_{1}^{-1}\left(C_{0}\right) \ldots\right) \cup \ldots f_{i+1}^{-1}\left(C_{i}\right) \cup C_{i+1}, f_{i+1}^{-1}\left(\ldots f_{1}^{-1}\left(O_{0}\right) \ldots\right)\right)
$$

establishing the case.

The result follows by induction.

Finally, we would like to put together these results into one result relating the initial machine $M_{0}$ to the final 

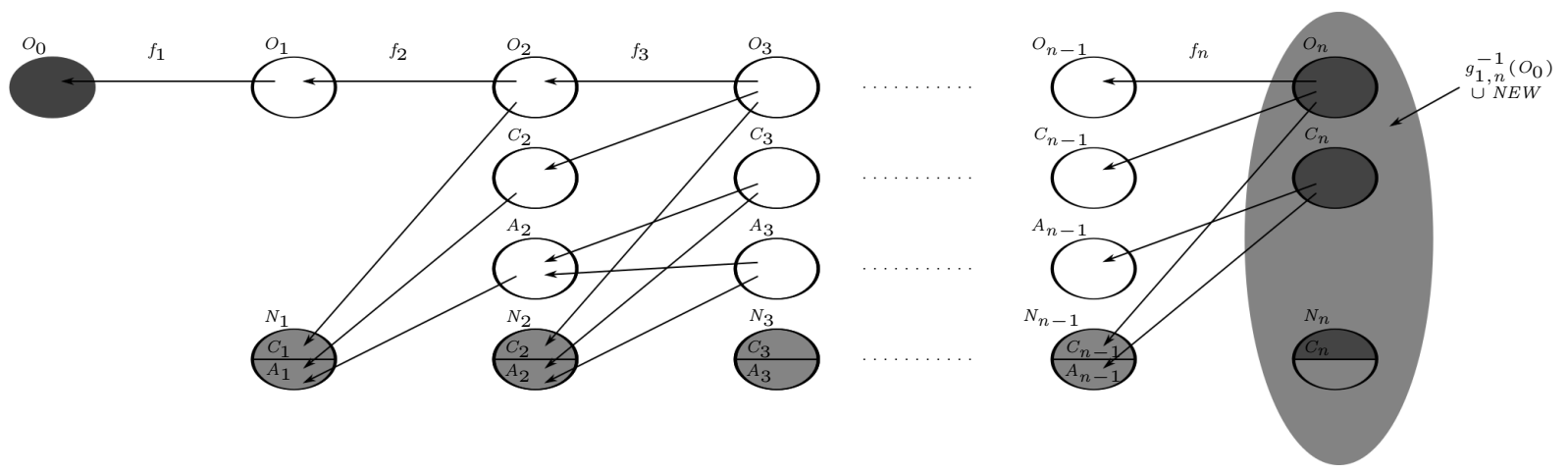

Fig. 9. Constructing $N E W$

machine $M_{n}$ in the refinement chain. This result should use hiding for the treatment of new events, and by stating the relationship between $M_{0}$ and $M_{n} \backslash\{$ new events $\}$ via infinite-traces-divergences refinement show that Event-B refinement actually does not introduce divergences on new events. This will be shown in Theorem 6.5 below. For such chains of refinement steps we have, by definition of strategy I, $A_{0}=C_{0}=\{\}$ (initially we have neither anticipated nor convergent events), and $A_{n}=\{\}$ (at the end all anticipated events have become convergent).

For this, we first of all need to find out what the "new events" are in the final machine. Define $g_{i, j}$ as the functional composition of the event mappings from $f_{j}$ to $f_{i}$ : (note that $g_{1,0}$ is the identity function)

$$
g_{i, j}=f_{j} ; f_{j-1} ; \ldots ; f_{i}
$$

Then by repeated application of

$$
C_{j} \cup A_{j} \cup O_{j}=f_{j}^{-1}\left(C_{j-1} \cup A_{j-1} \cup O_{j-1}\right) \cup N_{j}
$$

we obtain

$$
C_{j} \cup A_{j} \cup O_{j}=g_{1, j}^{-1}\left(C_{0} \cup A_{0} \cup O_{0}\right) \cup g_{2, j}^{-1}\left(N_{1}\right) \cup \ldots \cup g_{j, j}^{-1}\left(N_{j-1}\right) \cup N_{j}
$$

Also, by repeated application of

$$
O_{j}=f_{j}^{-1}\left(O_{j-1}\right) \cup f_{j}^{-1}\left(C_{j-1}\right)
$$

we obtain

$$
O_{j} \cup C_{j}=g_{1, j}^{-1}\left(O_{0}\right) \cup g_{1, j}^{-1}\left(C_{0}\right) \cup g_{2, j}^{-1}\left(C_{1}\right) \cup \ldots \cup g_{j, j}^{-1}\left(C_{j-1}\right) \cup C_{j}
$$

In a full refinement chain $M_{0} \preccurlyeq \ldots \preccurlyeq M_{n}$ we have, by the conditions of strategy I, that $A_{0}=\{\}, C_{0}=\{\}$, and $A_{n}=\{\}$.

Definition 6.3. The sets $N E W$ and $C O N$ are defined as follows:

$$
\begin{aligned}
N E W & =g_{2, n}^{-1}\left(N_{1}\right) \cup \ldots \cup g_{n, n}^{-1}\left(N_{n-1}\right) \cup N_{n} \\
C O N & =g_{2, n}^{-1}\left(C_{1}\right) \cup \ldots \cup g_{n, n}^{-1}\left(C_{n-1}\right) \cup C_{n}
\end{aligned}
$$

These constructions are illustrated in Figures 9 and 10.

Lemma 6.4. $N E W=C O N$

Proof From Equation 2 above with $j=n$, and using $A_{0}=C_{0}=A_{n}=\{\}$ we obtain

$$
C_{n} \cup O_{n}=g_{1, n}^{-1}\left(O_{0}\right) \cup N E W
$$

From Equation 3 above with $j=n$ we obtain

$$
C_{n} \cup O_{n}=g_{1, n}^{-1}\left(O_{0}\right) \cup C O N
$$




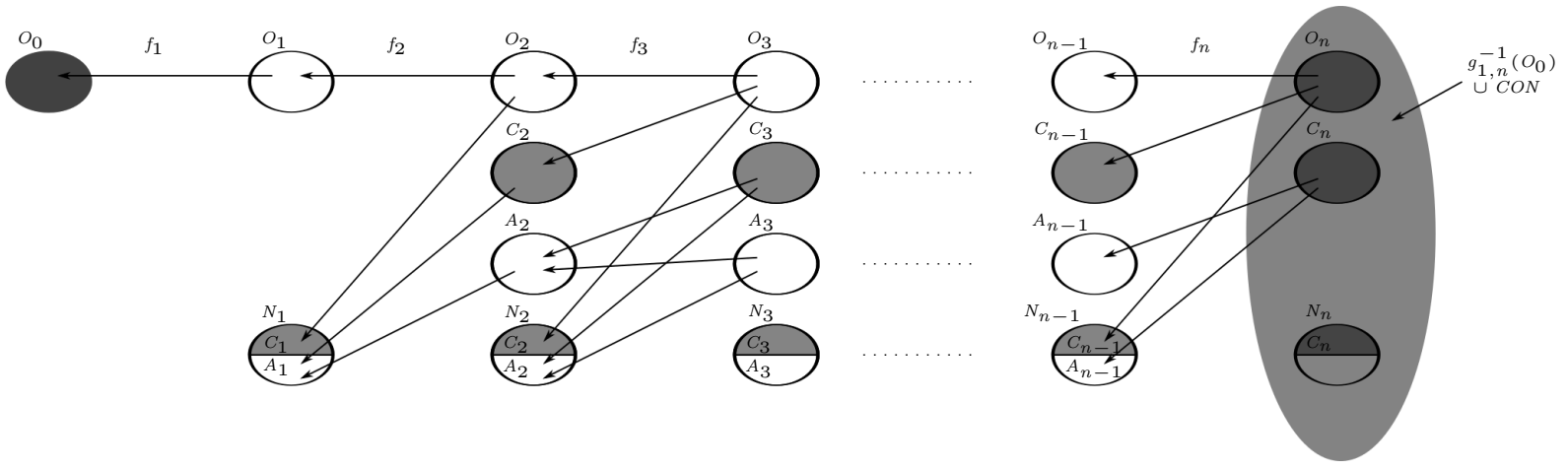

Fig. 10. Constructing $C O N$

Observe that $g_{1, n}^{-1}\left(O_{0}\right) \cap N E W=\{\}$ and $g_{1, n}^{-1}\left(O_{0}\right) \cap C O N=\{\}$, hence

$$
\left(C_{n} \cup O_{n}\right) \backslash g_{1, n}^{-1}\left(O_{0}\right)=N E W=C O N
$$

which yields the result.

From Theorems 5.2 and 6.2 above respectively we obtain that

$$
\begin{gathered}
f_{n}^{-1}\left(\ldots\left(f_{1}^{-1}\left(M_{0}\right)\right) \ldots\right)\|\| R U N_{N E W} \sqsubseteq_{T D I} M_{n} \\
\text { and } \quad M_{n} \text { sat } C A\left(C O N, f_{n}^{-1}\left(\ldots f_{1}^{-1}\left(O_{0}\right) \ldots\right)\right)
\end{gathered}
$$

Lemma 5.5(4) yields that $M_{n} \backslash C O N$ is divergence-free, i.e., $M_{n} \backslash N E W$ is divergence-free by Lemma 6.4. Hence by Lemma 4.1 we obtain that

$$
g_{1, n}^{-1}\left(M_{0}\right) \sqsubseteq_{T D I} M_{n} \backslash N E W
$$

or, equivalently, that the following theorem holds true.

Theorem 6.5. Let $M_{0} \preccurlyeq M_{1} \preccurlyeq \ldots \preccurlyeq M_{n}$ be a chain of refinement steps obeying strategy I and refining events according to functions $f_{i}$, and let $N E W$ be as defined in Definition 6.3. Then

$$
M_{0} \sqsubseteq_{T D I} g_{1, n}\left(M_{n} \backslash N E W\right)
$$

Proof: This follows from the result in Line 4 above, using the CSP law $f\left(f^{-1}(P)\right)=P$.

This result guarantees that Event-B refinement in strategy I neither introduces "new traces on old events", nor introduces divergences on new events. This gives us the precise account of Event-B refinement in terms of CSP.

Again, we make yet another extension of our example to demonstrate this result on a full refinement chain. Our final machine for strategy I shown in Figure 11 is Basket4.I. This machine is introducing a capacity for a basket and now allows adding only when the basket is not yet full. It furthermore makes event $a d d$, which has been anticipated so far, convergent. The corresponding variant to be shown to be decreased is $C A P$ - tot. Note that Basket4.I now has no anticipated events anymore and can thus serve as the final machine in our refinement chain. The function $f_{4 . I}$ is the identity (no renaming or splitting of events).

This completes the chain of refinements Basket $0 \preccurlyeq$ Basket $1 \preccurlyeq$ Basket $2 \preccurlyeq$ Basket $3 . I \preccurlyeq$ Basket4.I. Figure 12 shows the events appearing in the machines together with their status. By Theorem 6.5 we get our final result relating the CSP semantics of the initial with the final machine:

$$
\text { Basket } 0 \sqsubseteq_{T D I} f_{1}\left(f_{2}\left(f_{3 . I}\left(f_{4 . I}(\text { Basket } 4 . I \backslash\{a d d, \text { scan }\})\right)\right)\right)
$$

Following the non-extensible interface approach all newly introduced events have been made convergent and thus can safely be hidden without introducing divergence in the CSP semantics. 


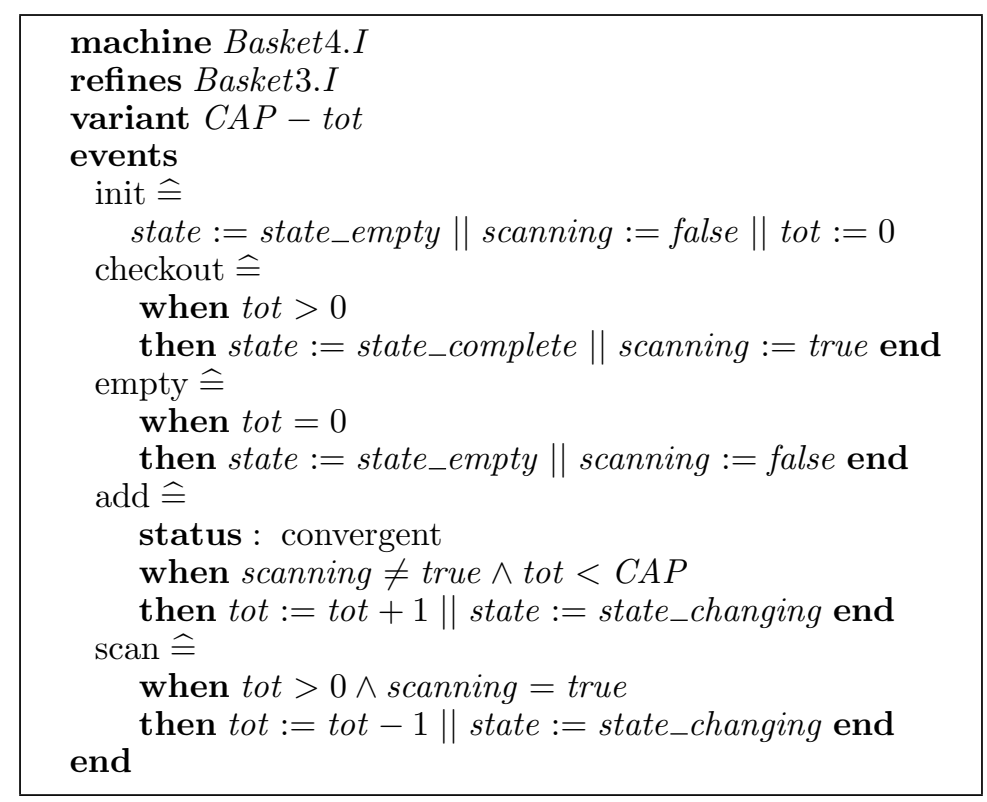

Fig. 11. Event-B machine Basket4.I

\begin{tabular}{|c|c|c|c|c|c|c|c|c|}
\hline \multirow{3}{*}{$\begin{array}{l}\text { Basket0 } \\
\text { checkout }(\mathrm{O}) \\
\text { empty }(\mathrm{O})\end{array}$} & \multirow{3}{*}{$\longleftarrow$} & \multirow{2}{*}{$\begin{array}{l}\text { Basket1 } \\
\text { checkout }(\mathrm{O})\end{array}$} & \multicolumn{2}{|c|}{ Basket2 } & \multicolumn{3}{|c|}{ Basket3.I } & \multirow{2}{*}{$\begin{array}{l}\text { Basket4.I } \\
\text { checkout }(\mathrm{O})\end{array}$} \\
\hline & & & $\longleftarrow$ & checkout $(\mathrm{O})$ & $\longleftarrow$ & checkout $(\mathrm{O})$ & $\longleftarrow$ & \\
\hline & & empty (O) & $\leftarrow$ & empty (O) & & empty (O) & $\leftarrow$ & empty (O) \\
\hline & & change (A) & $\leftarrow$ & add $(\mathrm{A})$ & $\leftarrow$ & add (A) & & add $(\mathrm{C})$ \\
\hline & & & & remove $(\mathrm{A})$ & & $\operatorname{scan}(\mathrm{C})$ & $\longleftarrow$ & $\operatorname{scan}(\mathrm{O})$ \\
\hline & $f_{1}$ & & $f_{2}$ & & $f_{3 . I}$ & & $f_{4 . I}$ & \\
\hline
\end{tabular}

Fig. 12. Machines and events in the development according to strategy I

\section{Strategy II: Interface Extension}

In this section we will look at the second development strategy which allows the interface of an Event-B machine to be extended. The main difference to the first strategy is that (a) new events can immediately have status ordinary, and (b) not all anticipated events need to be made convergent, but can also be made ordinary. For a development $M_{0} \preccurlyeq M_{1} \preccurlyeq \ldots \preccurlyeq M_{n}$ we thus get the following rules:

1. in the initial machine all events are ordinary;

2. each event of $M_{i}$ is refined by at least one event of $M_{i+1}$;

3. each new event in $M_{i}$ is either anticipated or convergent or ordinary;

4. each event in $M_{i+1}$ which refines an anticipated event of $M_{i}$ is itself either convergent or anticipated or ordinary;

5. refinements of convergent or ordinary events of $M_{i}$ are ordinary in $M_{i+1}$;

6. no anticipated events remain in the final machine.

The first as well as the last condition is just added to make reasoning about refinement relationships clearer. Both conditions impose no real restrictions on the development: given a sequence of machines which do not adhere to condition 1 and 6 , we can simply extend it with two new machines without getting additional proof obligations. The first condition can be met by introducing an extra initial machine with just the ordinary 


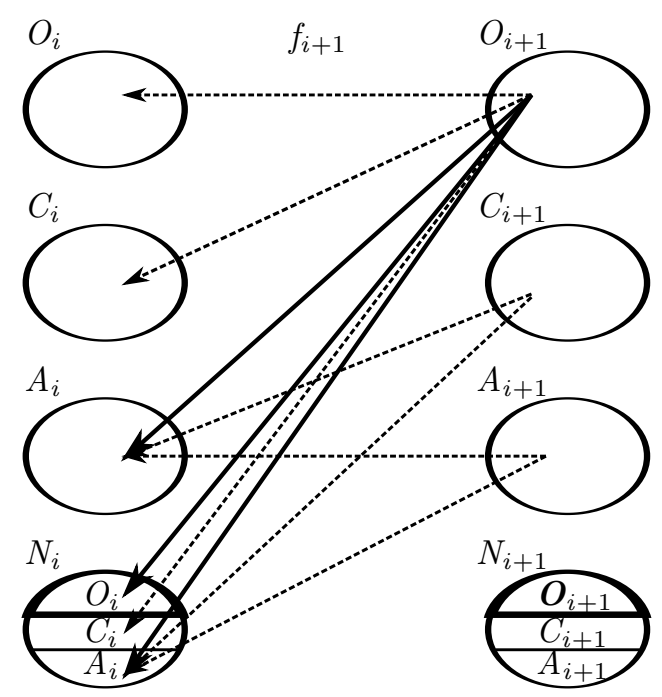

Fig. 13. Relationship between events in a refinement step in the extensible interface approach: $f_{i+1}$ maps events in $M_{i+1}$ to events in $M_{i}$ that they refine. The differences with the non-extensible interface approach are highlighted in bold.

events of the (previously initial) machine, and then in a first refinement steps introduce all the (initially wanted) anticipated and convergent events; the last condition can be similarly achieved by introducing an extra final machine which makes all anticipated events ordinary. Note also that some conditions do not impose any restrictions on refinements; they are merely stated here for getting a closer correspondence to the rules of strategy I. Again, we formalise the conditions.

1. $A_{0}=C_{0}=\{\}$;

2. $\operatorname{ran}\left(f_{i+1}\right)=A_{i} \cup C_{i} \cup O_{i}$;

3. $N_{i}=\left(A_{i} \cup C_{i} \cup O_{i}\right) \backslash \operatorname{dom}\left(f_{i}\right)$;

4. $f_{i+1}^{-1}\left(A_{i}\right) \subseteq C_{i+1} \cup A_{i+1} \cup O_{i+1}$;

5. $f_{i+1}^{-1}\left(C_{i} \cup O_{i}\right) \subseteq O_{i+1}$;

6. $A_{n}=\{\}$.

These relationships between the events are illustrated in Fig. 13. We observe that strategy II is the more flexible one. At each step in the refinement it allows the addition of new events, which (a) are added to the interface (new ordinary events), or (b) are added to the set of internal events (new convergent events) or for which the decision is deferred to later steps (anticipated events).

To see how this kind of development fits into a CSP refinement, we again calculate two sets of events introduced in a development. However, they play a different role this time. The first set, called $I F$, is the set of events which will be added during development as external events. Together with the ordinary events of the initial machine they constitute the interface of the system. Events are considered external when they are never shown to be convergent. We get additional external events when we have new events being ordinary, or new events being anticipated and never made convergent in the subsequent refinement steps. IF can be incrementally defined:

\section{Definition 7.1.}

$$
\begin{aligned}
I F_{0} & =\{\} \\
I F_{1} & =\left(f_{1}^{-1}\left(A_{0}\right) \cap O_{1}\right) \cup\left(N_{1} \cap O_{1}\right) \\
I F_{2} & =f_{2}^{-1}\left(I F_{1}\right) \cup\left(f_{2}^{-1}\left(A_{1}\right) \cap O_{2}\right) \cup\left(N_{2} \cap O_{2}\right) \\
& \ldots \\
I F_{j} & =f_{j}^{-1}\left(I F_{j-1}\right) \cup\left(f_{j}^{-1}\left(A_{j-1}\right) \cap O_{j}\right) \cup\left(N_{j} \cap O_{j}\right)
\end{aligned}
$$


Finally, $I F=I F_{n}$.

The second set of events is INT, the set of events added during development and considered internal in the final machine. Here, we find all those events which have been proven to be convergent in some of the development steps. Again, this set can be incrementally defined:

\section{Definition 7.2.}

$$
\begin{aligned}
I N T_{0} & =\{\} \\
I N T_{1} & =C_{1} \\
I N T_{2} & =f_{2}^{-1}\left(I N T_{1}\right) \cup C_{2} \\
& \cdots \\
I N T_{j} & =f_{j}^{-1}\left(I N T_{j-1}\right) \cup C_{j}
\end{aligned}
$$

Here, we get $I N T=I N T_{n}=g_{2, n}^{-1}\left(C_{1}\right) \cup g_{3, n}^{-1}\left(C_{2}\right) \cup \ldots \cup C_{n}$. The two sets of events $I F$ and $I N T$ are disjoint.

Lemma 7.3. Let $I F, I N T$ be the extended interface set and the set of internal events, respectively, as constructed in Definitions 7.1 and 7.2. Then

$$
I F \cap I N T=\{\} .
$$

Proof: We show this by induction on the number of steps in the development. More precisely, we show for every machine $M_{i}, 0 \leq i \leq n$, that $I N T_{i} \cap I F_{i}=\{\}$ (and $I N T_{i} \cap A_{i}=\{\}$ ). As a first observation note that $I F_{i} \subseteq O_{i}$.

Induction base Initially, both $I F_{0}$ and $I N T_{0}$ are empty as is $A_{0}$, thus all three sets are mutually disjoint. Induction step Assume $I N T_{i} \cap I F_{i}=\{\}, I N T_{i} \cap A_{i}=\{\}$ and $I F_{i} \cap A_{i}=\{\}$.

- Proof of $I N T_{i+1} \cap A_{i+1}=\{\}$. By definition $I N T_{i+1}=f_{i+1}^{-1}\left(I N T_{i}\right) \cup C_{i+1}$. The intersection of $A_{i+1}$ and $C_{i+1}$ is empty since every event can only have one status in a refinement step. Furthermore, $f_{i+1}^{-1}\left(I N T_{i}\right) \cap A_{i+1}=\{\}$ since $I N T_{i} \subseteq C_{i} \cup O_{i}$ and by rule 5 of the strategy none of these events can become anticipated.

- Proof of $I F_{i+1} \cap I N T_{i+1}=\{\}$. We have

$$
\begin{aligned}
& I F_{i+1}=f_{i+1}^{-1}\left(I F_{i}\right) \cup\left(f_{i+1}^{-1}\left(A_{i}\right) \cap O_{i+1}\right) \cup\left(N_{i+1} \cap O_{i+1}\right) \\
& I N T_{i+1}=f_{i+1}^{-1}\left(I N T_{i}\right) \cup C_{i+1}
\end{aligned}
$$

For their intersection we look at several cases: $(1) f_{i+1}^{-1}\left(I F_{i}\right) \cap f_{i+1}^{-1}\left(I N T_{i}\right)=\{\}$ follows from $f_{i+1}$ being a function and the induction hypothesis $I F_{i} \cap I N T_{i}=\{\} ;(2) f_{i+1}^{-1}\left(I F_{i}\right) \cap C_{i+1}=\{\}$ since $I F_{i} \subseteq O_{i}$, $f_{i+1}^{-1}\left(O_{i}\right) \subseteq O_{i+1}$ (rule 5 of the strategy) and $O_{i+1} \cap C_{i+1}=\{\} ;(3) f_{i+1}^{-1}\left(A_{i}\right) \cap O_{i+1} \cap C_{i+1}=\{\}$ since $O_{i+1} \cap C_{i+1}=\{\} ;(4) f_{i+1}^{-1}\left(A_{i}\right) \cap O_{i+1} \cap f_{i+1}^{-1}\left(I N T_{i}\right)=\{\}$ follows from $A_{i} \cap I N T_{i}=\{\}$ and $f_{i+1}$ being a function, and (5) $N_{i+1} \cap O_{i+1} \cap C_{i+1}=\{\}$ since the sets of ordinary and convergent events are disjoint, and finally (6) $N_{i+1} \cap O_{i+1} \cap f_{i+1}^{-1}\left(I N T_{i}\right)=\{\}$ since new and existing events are always different.

Note furthermore the event set $N E W$ constructed in the last section is the set $I N T$ : all newly introduced events are internal in strategy I. The set $I F$ would be empty in refinement chains of strategy I.

Next, we need to find out how this type of development fits into CSP refinement. We will see that the main difference to the first development strategy is that not all newly introduced events are made convergent but some are instead used to extend the interface. All convergent events can safely be hidden without introducing new divergences; all events in the interface extension can however only be tackled by the RUN process. Our relationship between the initial and final machine will thus take the form (renamings omitted):

$$
M_{0} \| \mid R U N_{I F} \sqsubseteq_{T D I} M_{n} \backslash I N T .
$$

Again, we first of all consider just two machines $M_{0}$ and $M_{1}$. Our first step is a generalisation of Lemma 4.1, which allows to split the events over the $R U N$ process into two sets, one of which is kept in $R U N$ and the other moved to the right hand side process and hidden. 
Lemma 7.4. IF $P_{0}\|\| R U N_{N_{1} \cup N_{2}} \sqsubseteq T D I P_{1}$ and $N_{1} \cap N_{2}=\{\}$ and $\left(N_{1} \cup N_{2}\right) \cap \alpha P_{0}=\{\}$ and $P_{1} \backslash N_{2}$ divergence-free, then $P_{0}|| R U N_{N_{1}} \sqsubseteq T D I P_{1} \backslash N_{2}$.

Proof: Assume that (1) $P_{0} \| \mid R U N_{N_{1} \cup N_{2}} \sqsubseteq_{T D I} P_{1}$ and (2) $N_{1} \cap N_{2}=\{\}$ and (3) $\left(N_{1} \cup N_{2}\right) \cap \alpha P_{0}=\{\}$ and (4) $P_{1} \backslash N_{2}$ divergence-free.

Traces Let $t r \in \operatorname{traces}\left(P_{1} \backslash N_{2}\right)$. By semantics of hiding there is some $t r^{\prime} \in \operatorname{traces}\left(P_{1}\right)$ such that $t r^{\prime} \backslash N_{2}=$ $t r$. By (1) $t r^{\prime} \in \operatorname{traces}\left(P_{0} \| R U N_{N_{1} \cup N_{2}}\right)$. By (2) and (3) $t r^{\prime} \backslash N_{2}=t r \in \operatorname{traces}\left(P_{0} \| \mid R U N_{N_{1}}\right)$.

Divergences By (3) divergences $\left(P_{1} \backslash N_{2}\right)=\{\}$, thus nothing to be proven here.

Infinites Let $u \in$ infinites $\left(P_{1} \backslash N_{2}\right)$. By semantics of hiding there is some $u^{\prime} \in$ infinites $\left(P_{1}\right)$ such that $u^{\prime} \backslash N_{2}=u$ and \# $\left(u^{\prime} \backslash N_{2}\right)=\infty$. By (1) $u^{\prime} \in$ infinites $\left(P_{0} \mid \| R U N_{N_{1} \cup N_{2}}\right)$. By (2) and (3) $u^{\prime} \backslash N_{2}=u \in$ infinites $\left(P_{0}|| \mid R U N_{N_{1}}\right)$.

This result can be used in a way similar to the usage of Lemma 4.1.

Lemma 7.5. Let $M_{0} \preccurlyeq M_{1}$ with events refined according to function $f_{1}$. Then

$$
f_{1}^{-1}\left(M_{0}\right) \||| R U N_{N_{1} \cap\left(A_{1} \cup O_{1}\right)} \sqsubseteq_{T D I} M_{1} \backslash\left(N_{1} \cap C_{1}\right)
$$

Proof: By Lemmas 5.5 (4) and 5.4 we get $M_{1} \backslash C_{1}$ divergence-free. By Lemma 5.1 we furthermore have $f_{1}^{-1}\left(M_{0}\right) \| R U N_{N_{1}} \sqsubseteq_{T D I} M_{1}$. We now distribute $N_{1}$ into disjoint partitions $N_{1} \cap\left(A_{1} \cup O_{1}\right)$ and $N_{1} \cap C_{1}$ and can now apply Lemma 7.4 which gives us the result.

Again similar to the previous section, we need a result about the $C A$-predicate in refinement chains constructed according to strategy II. The following lemma can be seen as the generalisation of Lemma 5.6 to strategy II. This time we have to take into account that anticipated events can be refined straight into ordinary events during a refinement step, and also ordinary events can be introduced as new events in a refinement step. Hence the $O^{\prime}$ referred to in the $C A$ predicate which the refinement machine needs to satisfy must also take these new sets into account.

Lemma 7.6. Let $M \preccurlyeq M^{\prime}$ be constructed according to strategy II with an associated refinement function $f^{\prime}$ and let $M$ sat $C A(C, O)$. Then $M^{\prime}$ sat $C A\left(f^{\prime-1}(C) \cup C^{\prime}, f^{\prime-1}(O) \cup\left(f^{\prime-1}(A) \cap O^{\prime}\right) \cup\left(N^{\prime} \cap O^{\prime}\right)\right)$.

Proof: Assume $u \in \operatorname{infinites}\left(M^{\prime}\right)$ and \# $\left(u \uparrow\left(f^{\prime-1}(C) \cup C^{\prime}\right)\right)=\infty$. We aim to establish that \# $(u \uparrow$ $\left.f^{\prime-1}(O) \cup\left(f^{\prime-1}(A) \cap O^{\prime}\right) \cup\left(N^{\prime} \cap O^{\prime}\right)\right)=\infty$. We have $\#\left(u\left\lceil f^{\prime-1}(C)\right)=\infty\right.$ or $\#\left(u\left\lceil C^{\prime}\right)=\infty\right.$.

In the former case, Lemma 5.1 yields that $f^{\prime}\left(u\left\lceil f^{-1}(\alpha M)\right) \in\right.$ infinites $(M)$. Then

$$
\begin{array}{rlrl}
\#\left(u\left\lceil f^{\prime-1}(C)\right)=\infty\right. & & \text { (given) } \\
\#\left(f^{\prime}\left(u\left\lceil f^{\prime-1}(C)\right) \uparrow C\right)=\infty\right. & \text { (since renaming preserves length) } \\
\#\left(f^{\prime}\left(u\left\lceil f^{\prime-1}(\alpha M)\right) \uparrow C\right)=\infty\right. & \text { (since } C \subseteq \alpha M) \\
\#\left(f^{\prime}\left(u\left\lceil f^{\prime-1}(\alpha M)\right) \uparrow O\right)=\infty\right. & \text { (by } M \text { sat } C A(C, O)) \\
\#\left(u\left\lceil f^{\prime-1}(\alpha M)\right) \uparrow f^{\prime-1}(O)=\infty\right. & \text { (since renaming preserves length) } \\
\#\left(u\left\lceil f^{\prime-1}(O)\right)=\infty\right. & \text { (since } O \subseteq \alpha M)
\end{array}
$$

In the latter case Lemma 5.4 yields that $\#\left(u\left\lceil O^{\prime}\right)=\infty\right.$. Then we have $\#\left(u\left\lceil f^{\prime-1}(C \cup O) \cup\left(f^{\prime-1}(A) \cap\right.\right.\right.$ $\left.\left.O^{\prime}\right) \cup\left(N^{\prime} \cap O^{\prime}\right)\right)=\infty$ (since $O^{\prime}$ is by definition of strategy II $\left.f^{\prime-1}(C \cup O) \cup\left(f^{\prime-1}(A) \cap O^{\prime}\right) \cup\left(N^{\prime} \cap O^{\prime}\right)\right)$. Hence $\#\left(u\left\lceil f^{\prime-1}(C)\right)=\infty\right.$ or $\#\left(u\left\lceil f^{\prime-1}(O)\right)=\infty\right.$ or $\#\left(u\left\lceil f^{\prime-1}(A) \cap O^{\prime}\right)=\infty\right.$ or $\#\left(u\left\lceil\left(N^{\prime} \cap O^{\prime}\right)\right)=\infty\right.$. The second, third and fourth cases make up the desired result, the first is the case already treated above.

For our next theorem we first require a lemma:

Lemma 7.7. If $M_{0} \preccurlyeq M_{1} \preccurlyeq \ldots \preccurlyeq M_{n}$ then for each $i \leqslant n$ :

$$
O_{i}=f_{i}^{-1}\left(I N T_{i-1}\right) \cup I F_{i} \cup g_{1, i}^{-1}\left(O_{0}\right)
$$

Proof: By induction on $n$.

Induction base Assume $n=0$. This case reduces to $O_{0}=O_{0}$, which establishes the case. 
Induction step Assume the result for $i$, we aim to establish it for $i+1$. The inductive hypothesis gives:

$$
O_{i}=f_{i}^{-1}\left(I N T_{i-1}\right) \cup I F_{i} \cup g_{1, i}^{-1}\left(O_{0}\right)
$$

We have

$$
\begin{aligned}
O_{i+1} & =\left(f_{i+1}^{-1}\left(O_{i} \cup C_{i} \cup A_{i}\right) \cap O_{i+1}\right) \cup\left(N_{i} \cap O_{i}\right) \\
& =f_{i+1}^{-1}\left(O_{i}\right) \cup f_{i+1}^{-1}\left(C_{i}\right) \cup f_{i+1}^{-1}\left(A_{i}\right) \cap O_{i+1} \cup\left(N_{i+1} \cap O_{i+1}\right) \\
& =f_{i+1}^{-1}\left(f_{i}^{-1}\left(I N T_{i-1}\right) \cup I F_{i} \cup g_{1, i}^{-1}\left(O_{0}\right)\right) \cup f_{i+1}^{-1}\left(C_{i}\right) \cup f_{i+1}^{-1}\left(A_{i}\right) \cap O_{i+1} \cup\left(N_{i+1} \cap O_{i+1}\right) \\
& =f_{i+1}^{-1}\left(( f _ { i } ^ { - 1 } ( I N T _ { i - 1 } ) \cup C _ { i } ) \cup \left(f_{i+1}^{-1}\left(I F_{i}\right) \cup f_{i+1}^{-1}\left(A_{i}\right) \cap O_{i+1} \cup\left(N_{i+1} \cap O_{i+1}\right) \cup\left(f_{i+1}^{-1}\left(g_{1, i}^{-1}\left(O_{0}\right)\right)\right)\right.\right. \\
& =f_{i+1}^{-1}\left(I N T_{i}\right) \cup I F_{i+1} \cup g_{1, i+1}^{-1}\left(O_{0}\right)
\end{aligned}
$$

which establishes the case.

We now obtain the following result on refinement chains:

Theorem 7.8. If $M_{0} \preccurlyeq M_{1} \preccurlyeq \ldots \preccurlyeq M_{n}$ then

$M_{n}$ sat $C A\left(I N T_{n}, g_{1, n}^{-1}\left(O_{0}\right) \cup I F_{n}\right)$

Proof: By induction on $n$.

Induction base Assume $n=0$. In this case $I N T_{0}=\{\}$, and $g_{1,0}^{-1}\left(O_{0}\right) \cup I F_{0}=O_{0}$. The result immediately follows since $g_{1,0}$ is the identify, and $M_{0}$ sat $C A\left(\{\}, O_{0}\right)$ holds vacuously, establishing the case.

Induction step Assume the result for $i$, we aim to establish it for $i+1$. The inductive hypothesis gives:

$$
M_{i} \text { sat } C A\left(I N T_{i}, g_{1, i}^{-1}\left(O_{0}\right) \cup I F_{i}\right)
$$

and from Lemma 5.4 we have:

$$
M_{i+1} \text { sat } C A\left(C_{i+1}, O_{i+1}\right)
$$

Now for $M_{i}=M$ and $M_{i+1}=M^{\prime}$ we define

$$
\begin{aligned}
C & =I N T_{i} \\
O & =g_{1, i}^{-1}\left(O_{0}\right) \cup I F_{i} \\
C^{\prime} & =C_{i+1} \\
O^{\prime} & =O_{i+1} \\
X & =\left(f_{i+1}^{-1}\left(A_{i}\right) \cap O_{i+1}\right) \cup\left(N_{i+1} \cap O_{i+1}\right)
\end{aligned}
$$

Now

$$
\begin{array}{rlr}
O^{\prime} & =O_{i+1} & \\
& =\left(f_{i+1}^{-1}\left(O_{i} \cup C_{i} \cup A_{i}\right) \cap O_{i+1}\right) \cup\left(N_{i+1} \cap O_{i+1}\right) & \\
& =f_{i+1}^{-1}\left(O_{i}\right) \cup f_{i+1}^{-1}\left(C_{i}\right) \cup\left(\left(f_{i+1}^{-1}\left(A_{i}\right) \cap O_{i+1}\right) \cup\left(N_{i+1} \cap O_{i+1}\right)\right) & \quad \text { (by Lemma } 7.7) \\
& =f_{i+1}^{-1}\left(f_{i}^{-1}\left(I N T_{i-1}\right) \cup I F_{i} \cup g_{1, i}^{-1}\left(O_{0}\right)\right) \cup f_{i+1}^{-1}\left(C_{i}\right) \cup X & \\
& =f_{i+1}^{-1}\left(f_{i}^{-1}\left(I N T_{i-1}\right) \cup f_{i+1}^{-1}\left(I F_{i}\right) \cup f_{i+1}^{-1}\left(g_{1, i}^{-1}\left(O_{0}\right)\right) \cup f_{i+1}^{-1}\left(C_{i}\right) \cup X\right. & \\
& =f_{i+1}^{-1}\left(f_{i}^{-1}\left(I N T_{i-1}\right)\right) \cup f_{i+1}^{-1}\left(C_{i}\right) \cup f_{i+1}^{-1}\left(I F_{i}\right) \cup f_{i+1}^{-1}\left(g_{1, i}^{-1}\left(O_{0}\right)\right) \cup X & \quad \text { (reordering terms) } \\
& =f_{i+1}^{-1}\left(I N T_{i}\right) \cup f_{i+1}^{-1}\left(I F_{i} \cup\left(g_{1, i}^{-1}\left(O_{0}\right)\right)\right) \cup X & \\
& =f_{i+1}^{-1}(C) \cup f_{i+1}^{-1}(O) \cup X &
\end{array}
$$

It follows from Lemma 5.6 that

$$
M_{i+1} \text { sat } C A\left(f_{i+1}^{-1}\left(I N T_{i}\right) \cup C_{i+1}, f_{i+1}^{-1}\left(g_{1, i}^{-1}\left(O_{0}\right) \cup I F_{i}\right) \cup\left(f_{i+1}^{-1}\left(A_{i}\right) \cap O_{i+1}\right) \cup\left(N_{i+1} \cap O_{i+1}\right)\right)
$$

By the definitions of $I N T_{i+1}$ and $I F_{i+1}$ this reduces to

$$
M_{i} \text { sat } C A\left(I N T_{i+1}, g_{1, i+1}^{-1}\left(O_{0}\right) \cup I F_{i+1}\right)
$$




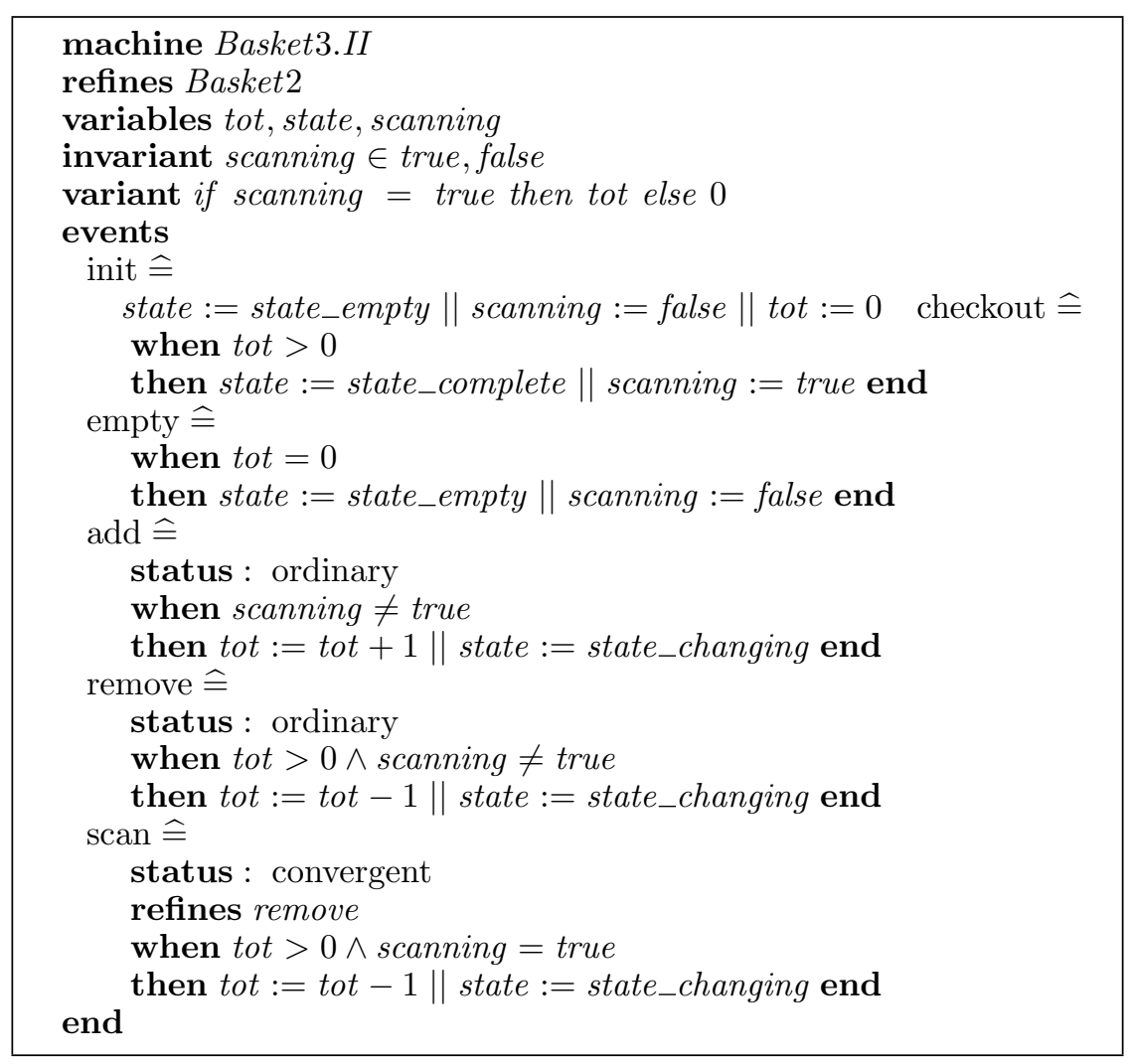

Fig. 14. Alternative development of the basket following approach 2

which establishes the case.

The result follows by induction.

Since $I N T \cap\left(g_{2, n}\left(O_{0}\right) \cup I F\right)=\{\}$ we furthermore get $M_{n} \backslash I N T$ divergence-free. Together with Lemma 7.4 we finally obtain

Theorem 7.9. Let $M_{0} \preccurlyeq M_{1} \preccurlyeq \ldots \preccurlyeq M_{n}$ be a chain of refinement steps following strategy II, refining events according to functions $f_{i}$, and let $I F$ and $I N T$ be the two sets calculated above. Then

$$
g_{1, n}^{-1}\left(M_{0}\right) \| R U N_{I F} \sqsubseteq T D I M_{n} \backslash I N T
$$

In Figure 14 we see an alternative development of Basket2 following this second development strategy. Instead of making the anticipated event add of Basket 2 convergent (or keep it anticipated), we put it into the interface and make it ordinary. The event remove of Basket 2 is now split into an ordinary event remove and a convergent event scan. The machine thus ends up with having an interface \{checkout, empty, add, remove $\}$ while having only one internal event which is scan. Thus for the development Basket $0 \preccurlyeq$ Basket $1 \preccurlyeq$ Basket $2 \preccurlyeq$ Basket3.II with event sets and event status as shown in Figure 15 we obtain $I F=\{$ add, remove $\}$ (the extension of the interface) and $I N T=\{s c a n\}$, and thus by Theorem 7.9 we get

$$
f_{3 . I I}^{-1}\left(f_{2}^{-1}\left(f_{1}^{-1}(\text { Basket } 0)\right)\right)\|\| R U N_{\{\text {add,remove }\}} \sqsubseteq T D I \text { Basket } 3 . I I \backslash\{\text { scan }\}
$$

For example, one trace of Basket3.II \\{scan\} is the trace

$$
\langle a d d, \text { add, remove, checkout, scan, empty }\rangle \backslash\{\text { scan }\}=\langle\text { add, add, remove, checkout, empty }\rangle
$$

This can be separated out into

$$
\langle\text { checkout, empty }\rangle \in \operatorname{traces}\left(f_{3 . I I}^{-1}\left(f_{2}^{-1}\left(f_{1}^{-1}(\text { Basket } 0)\right)\right)\right)
$$




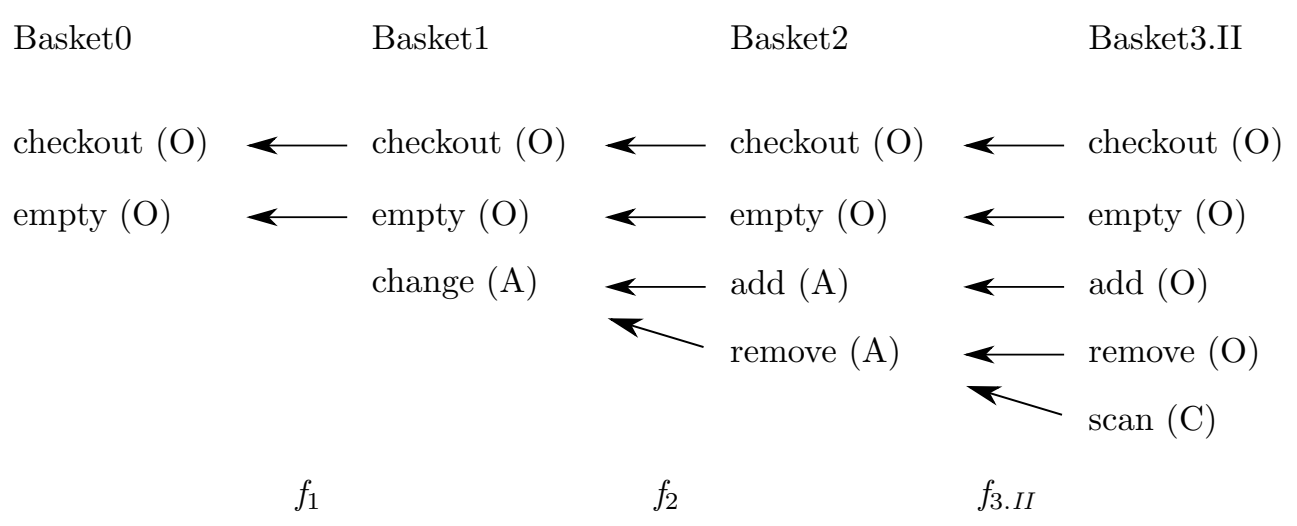

Fig. 15. Machines and events in the development according to strategy II

and

$\langle a d d$, add, remove $\rangle \in \operatorname{traces}\left(R U N_{\{\text {add,remove }\}}\right)$

This shows that the trace is also a trace of the interleaving of these two component processes, $f_{3 . I I}^{-1}\left(f_{2}^{-1}\left(f_{1}^{-1}(\right.\right.$ Basket 0$\left.\left.)\right)\right) \mid \| R U N_{\{a d d, \text { remove }\}}$, in line with Theorem 7.9.

This completes the basket example for which we now have two different developments and the corresponding results in terms of CSP refinement.

\section{Conclusion}

In this paper, we have given a behavioural semantics account of Event-B refinement. The approach builds on Butler's semantics for action systems [But92]. Butler's refinement rules allow new convergent events to be introduced into action systems, so that a refinement step from machine $M_{i}$ to machine $M_{i+1}$, introducing new events $N_{i+1}$, satisfies $M_{i} \sqsubseteq T D I\left(M_{i+1} \backslash N_{i+1}\right)$, thus ensuring that hiding the new events does not introduce divergence. Abrial's approach to Event-B refinement [Abr10] generalises this approach, allowing new events to be anticipated (deferring making it internal) as well as convergent (to be considered as internal), and also allowing splitting of events. The approach requires that anticipated events become convergent at some refinement step, so that all events introduced during refinement are shown at some point to be convergent. This corresponds to strategy I described in Section 6. The Rodin tool for Event-B [BH07, EB11, ABH $\left.{ }^{+} 10\right]$ generalises Abrial's approach in supporting a more liberal treatment of anticipated events, and does not require that they are eventually made convergent but allows them to be added to the interface instead. In this approach treatment of an event as anticipated therefore defers the decision as to whether it will ultimately be internal or external. This is strategy II as described in Section 7. Rodin does have some superficial differences with strategy II, notably that it allows refinements of convergent events to also be labelled as convergent, whereas strategy II requires them to be ordinary. In fact they are treated as ordinary by Rodin, since the proof obligations generated do not require them to decrease the variant. Rodin allows such events to be labelled as convergent to allow the developer to record that they were shown to be convergent at some stage.

Our approach to refinement using CSP semantics reflects the introduction of anticipated events and splitting, and thus extends Butler's approach, in order to encompass these different forms of event treatment in Event-B refinement. We obtain a clear statement of the relationship between the first and the last machines in a refinement chain, which follows from the transitivity of refinement along the chain, together with the additional requirements on the first and last machines: that the first should contain only ordinary events, and that the last should not contain any anticipated events. For strategy I the relationship is given by Theorem 6.5, and for strategy II by Theorem 7.9. We do not yet handle merging events, and this is the subject of current research. The handling of divergence in refinement, though not in an Event-B setting, has also been investigated in [BD09]. Proofs of divergence freedom for particular events also come into play when studying liveness properties of Event-B machines. This has recently been investigated in [HA11], looking at properties specified in temporal logic. 
Recently, an Event-B $\|$ CSP approach has been introduced [STW10]. It aims to combine Event-B machine descriptions with CSP [Sch99] control processes, in order to support a more explicit view of control. In this, it follows previous works on integration of formal methods [But00, WC02, DS03, OW05, ST05, Ili09], which aim at complementing a state-based specification formalism with a process algebra.

The account of refinement presented here provides the basis for a flexible refinement framework in Event$\mathrm{B} \| \mathrm{CSP}$, and this is presented in [STW11c]. The semantics justifies the introduction of a new status of devolved, for refinement events which are anticipated in the Event-B machine but convergent in the CSP controller. This approach has been applied to an initial Event-B $\|$ CSP case study of a Bounded Retransmission Protocol [STW11a]. We aim to investigate further case studies. We are in particular interested in finding out whether the work of showing divergence-freedom (and also deadlock-freedom) can be divided onto the Event-B and CSP part such that for some events convergence is guaranteed by showing the corresponding proof obligations in Event-B while for others we just look at divergence-freedom of the CSP process. The latter part could then be supported by model checking tools for CSP, like FDR [For]. Another strand of future work would be an investigation of decomposition techniques for Event-B [But09, HA10, SHWI11] and their impact on the CSP semantics.

Acknowledgement. Many thanks to Thai Son Hoang for numerous discussions on Event-B development strategies. Thanks also to the anonymous referees for their careful reading of the paper and for their constructive suggestions.

\section{References}

$\left[\mathrm{ABH}^{+} 10\right] \quad J-R$. Abrial, M. J. Butler, S. Hallerstede, T. S. Hoang, F. Mehta, and L. Voisin. Rodin: an open toolset for modelling and reasoning in Event-B. STTT, 12(6):447-466, 2010.

[ABHV08] J-R. Abrial, M. J. Butler, S. Hallerstede, and L. Voisin. A roadmap for the Rodin toolset. In E. Börger, M. J. Butler, J. P. Bowen, and P. Boca, editors, ABZ, volume 5238 of Lecture Notes in Computer Science, page 347. Springer, 2008.

[Abr05] J-R. Abrial. The B-book - assigning programs to meanings. Cambridge University Press, 2005.

[Abr10] J-R. Abrial. Modeling in Event-B: System and Software Engineering. Cambridge University Press, 2010.

[BD02] C. Bolton and J. Davies. Refinement in Object-Z and CSP. In M. Butler, L. Petre, and K. Sere, editors, IFM 2002: Integrated Formal Methods, number 2335 in LNCS, pages 225-244, 2002.

[BD09] E. A. Boiten and J. Derrick. Modelling divergence in relational concurrent refinement. In Leuschel and Wehrheim [LW09], pages 183-199.

[BH07] M. J. Butler and S. Hallerstede. The Rodin formal modelling tool. In BCS-FACS Christmas 2007 Meeting Formal Methods In Industry, 2007.

[But92] M. J. Butler. A CSP approach to Action Systems. DPhil thesis, Oxford University, 1992.

[But00] M. J. Butler. csp2B: A practical approach to combining CSP and B. In FACS, pages 182-196, 2000.

[But09] M. J. Butler. Decomposition structures for Event-B. In Leuschel and Wehrheim [LW09], pages 20-38.

[But12] M. J. Butler. External and internal choice with event groups in Event-B. Formal Aspects of Computing, 24(46):555-567, 2012.

[BvW98] R-J. Back and J. von Wright. Refinement Calculus: A Systematic Introduction. Graduate Texts in Computer Science. Springer, 1998.

[DB01] J. Derrick and E.A. Boiten. Refinement in $Z$ and Object-Z. Springer-Verlag, 2001.

[DB03] J. Derrick and E.A. Boiten. Relational concurrent refinement. Formal Aspects of Computing, 15(2-3):182-214, November 2003.

[DS03] J. Derrick and G. Smith. Structural refinement of systems specified in Object-Z and CSP. Formal Asp. Comput., 15(1):1-27, 2003.

[EB11] Event-B.org. Rodin platform version 2.2.2, release date 2011/06/01. http://www. event-b.org/, 2011.

[For] Formal Systems (Europe) Ltd. The FDR model checker. http://www.fsel.com/ (accessed 8/3/11).

[HA10] T.S. Hoang and J-R. Abrial. Event-B decomposition for parallel programs. In M. Frappier, U. Glässer, S. Khurshid, R. Laleau, and S. Reeves, editors, ABZ, volume 5977 of Lecture Notes in Computer Science, pages 319-333. Springer, 2010.

[HA11] T.S. Hoang and J-R. Abrial. Reasoning about liveness properties in Event-B. In Shengchao Qin and Zongyan Qiu, editors, ICFEM, volume 6991 of Lecture Notes in Computer Science, pages 456-471. Springer, 2011.

[Hal11] Stefan Hallerstede. On the purpose of Event-B proof obligations. Formal Asp. Comput., 23(1):133-150, 2011.

[Hoa85] C.A.R. Hoare. Communicating Sequential Processes. Prentice-Hall, 1985.

[Ili09] A. Iliasov. On Event-B and control flow. Technical Report CS-TR-1159, School of Computing Science, Newcastle University, August 2009.

[Jac02] D. Jackson. Alloy: a lightweight object modelling notation. ACM Trans. Softw. Eng. Methodol., 11(2):256-290, 2002 .

[LW09] M. Leuschel and H. Wehrheim, editors. Integrated Formal Methods, 7th International Conference, IFM 2009, Düsseldorf, Germany, February 16-19, 2009. Proceedings, volume 5423 of Lecture Notes in Computer Science. Springer, 2009. 
[MAV05] C. Métayer, J.-R. Abrial, and L. Voisin. Event-B language, 2005. RODIN Project Deliverable 3.2, http://rodin.cs.ncl.ac.uk/deliverables/D7.pdf, accessed 25/5/10.

[Mor88] C.C. Morgan. The specification statement. ACM Trans. Program. Lang. Syst., 10(3):403-419, 1988.

[Mor90] C.C. Morgan. Of wp and CSP. Beauty is our business: a birthday salute to E. W. Dijkstra, pages 319-326, 1990.

[OW05] E-R. Olderog and H. Wehrheim. Specification and (property) inheritance in CSP-OZ. Sci. Comput. Program., $55(1-3): 227-257,2005$

[Ros98] A.W. Roscoe. Theory and Practice of Concurrency. Prentice-Hall, 1998.

[Sch99] S. Schneider. Concurrent and Real-time Systems: The CSP approach. Wiley, 1999.

[SHWI11] R.A. Silva, T.S. Hoang, W. Wei, and A. Iliasov. A survey on Event-B decomposition. In Workshop on Automated Verification of Critical Systems (AVOCS 2011), 2011.

[ST05] S. Schneider and H. Treharne. CSP theorems for communicating B machines. Formal Asp. Comput., 17(4):390-422, 2005.

[STW10] S. Schneider, H. Treharne, and H. Wehrheim. A CSP approach to control in Event-B. In IFM, pages 260-274, 2010 .

[STW11a] S. Schneider, H. Treharne, and H. Wehrheim. Bounded retransmission in Event-B\|CSP: a case study. In Workshop B 2011, ENTCS, 2011.

[STW11b] S. Schneider, H. Treharne, and H. Wehrheim. A CSP account of Event-B refinement. In J. Derrick, E.A. Boiten, and S. Reeves, editors, Refine 2011, volume 55 of EPTCS, pages 139-154, 2011.

[STW11c] S. Schneider, H. Treharne, and H. Wehrheim. Stepwise refinement in Event-B $\|$ CSP. Technical Report CS-11-03, University of Surrey, 2011.

[WC02] J. C. P. Woodcock and A. Cavalcanti. The semantics of circus. In D. Bert, J. P. Bowen, M. C. Henson, and K. Robinson, editors, ZB 2002, volume 2272 of Lecture Notes in Computer Science, pages 184-203. Springer, 2002.

[WD96] J. C. P. Woodcock and J. Davies. Using Z: Specification, Refinement, and Proof. Prentice Hall, 1996. 